\title{
Analyse \\ du fonctionnement d'un groupe bulbe grâce à des prospections de l'écoulement en amont et en aval de la roue
}

\author{
Operational analysis of a bulb unit \\ from flow measurements \\ upstream and downstream of the runner
}

\author{
PAR P. CAMPMAS
}

\begin{abstract}
CHEF DU SERVICE ÉTUDES ET ESSAIS SPÉCIAUX A LA DIVISION TECHNYQUE GX́NÉRALE, SERVICE DE LA PRODUCTION HYDRAULIQUE D'ÉLECTRICITÉ DE FHANGE, A GRENOHLE

Communication présentée à la séance du 16 mars 1961

du Comité Technique de la Société Hydrotechnique de France
\end{abstract}

\begin{abstract}
Bien que des mesures absolues et même relalives du rendement par les méthodes classiques se soient révélées impossibles, l'analyse assez complète du fonctionnement d'un groupe bulbe de $9 \mathrm{MW}$ a pu être effectuée grâce à une prospection de l'écoulement en amont et en aval de la roue.

Brève description du dispositif expérimental.

Application des résultats obtenus a l'étude:

- du rendement et de la conjugaison optimale entre pales et vannage;

- des angles moyens d'entrée;

- de la symétrie de l'écoulement;

- de la localisation des pertes dans la turbine.

Indications sur le détail de l'éconlement.

Les résultats obtenus justifient la poursuite de telles investigations et l'aménagement, sur les grosses installations en construction, des accès nécessaires d̀ l'exécution d'essais dans des conditions satisfaisantes.
\end{abstract}

\begin{abstract}
Despite the fact that absolute-or even relative -efficiency measurements have proved impossible to carry out by conventional methods, a fairly complete operational analysis has been successfully performed on a $9 \mathrm{MW}$ bulb unit b!y investigating the flows upstream and downstream of the runner.

Brief description of the experimental equipment used.

Application of the results to studies of the followving:

1) Efficiency and oplimum, guide pune setting combination;

2) Mean entry angles;

3) Flow symmelry;

4) Localisation of losses in the turbine. Detailed indications on the flow pattern.

The results obtained justify further investigalions of this type and the provision of major installations under construction with the necessary means of access for the carrying out of such measurements under satisfactory conditions.
\end{abstract}

Caractéristiques de la turbine

\begin{tabular}{|l|c|c|c|}
\hline Hauteur de chute ........ & $12,5 \mathrm{~m}$ & $11 \mathrm{~m}$ & $9,85 \mathrm{~m}$ \\
Puissance $\ldots \ldots \ldots \ldots \ldots \ldots \ldots$ & $9,1 \mathrm{MW}$ & $9,1 \mathrm{MW}$ & $7,8 \mathrm{MW}$ \\
Vitesse $\ldots \ldots \ldots \ldots \ldots \ldots \ldots$ & $150 \mathrm{tr} / \mathrm{mn}$ & & \\
\hline
\end{tabular}




\section{INTRODUCTION}

Il est souvent difficile de mesurer la valeur absolue du rendement des turbines de basse chute mais, pour les Kaplan classiques, il est toujours possible d'effectuer des mesures relatives (qui permettent en particulier de définir ou de vérifier la conjugaison optimale entre pales et vannage) grâce à des contròles pićzométriques du débit installés dans la bâche.

Cette disposition n'est pas forcément transposable sur les groupes bulbes; et, effectivement, pour une machine de ce type $(9 \mathrm{MW}$ sous $11 \mathrm{~m}$ de chute à $150 \mathrm{tr} / \mathrm{mn}$ ), les procédés classiques de mesures absolues ou relatives du rendement se sont révélés inapplicables.
Le seul contrôle industriel réalisable sans trop de difficultés était une prospection de l'écoulement au voisinage de la roue où l'on disposait des accès nécessaires. Entrepris d'abord dans l'aspirateur avec des objectifs limités, cet essai se révélait rapidement très fructueux. Il était alors complété par des sondages entre distributeur et roue et permettait, non seulement la détermination de la conjugaison optimale et une évaluation du rendement, mais encore une analyse relativement complète et détaillée du fonctionnement de la turbine.

\section{CONDITIONS EXPÉRIMENTALES}

\section{Prospection de l'écoulement à la sortie de la roue.}

Elle a été réalisée avec un tube de Pitot placé successivement sur deux rayons perpendiculaires, inclinés à $45^{\circ}$ sur la verticale et situés à $1,2 \mathrm{~m}$ de l'axe des pales (fig. 1, diamètre de la roue $3,8 \mathrm{~m}$ ).
La technique d'essai. couramment utilisée depuis plusieurs années, peut être considérée comme classique (1). Rappelons simplement qu'on détermine en divers points du rayon de mesure la pression et la vitesse de l'eau, en

(1) Les premières mesures de ce type, dues à M. Fontaine, ont été exécutées à Brommat en 1951.

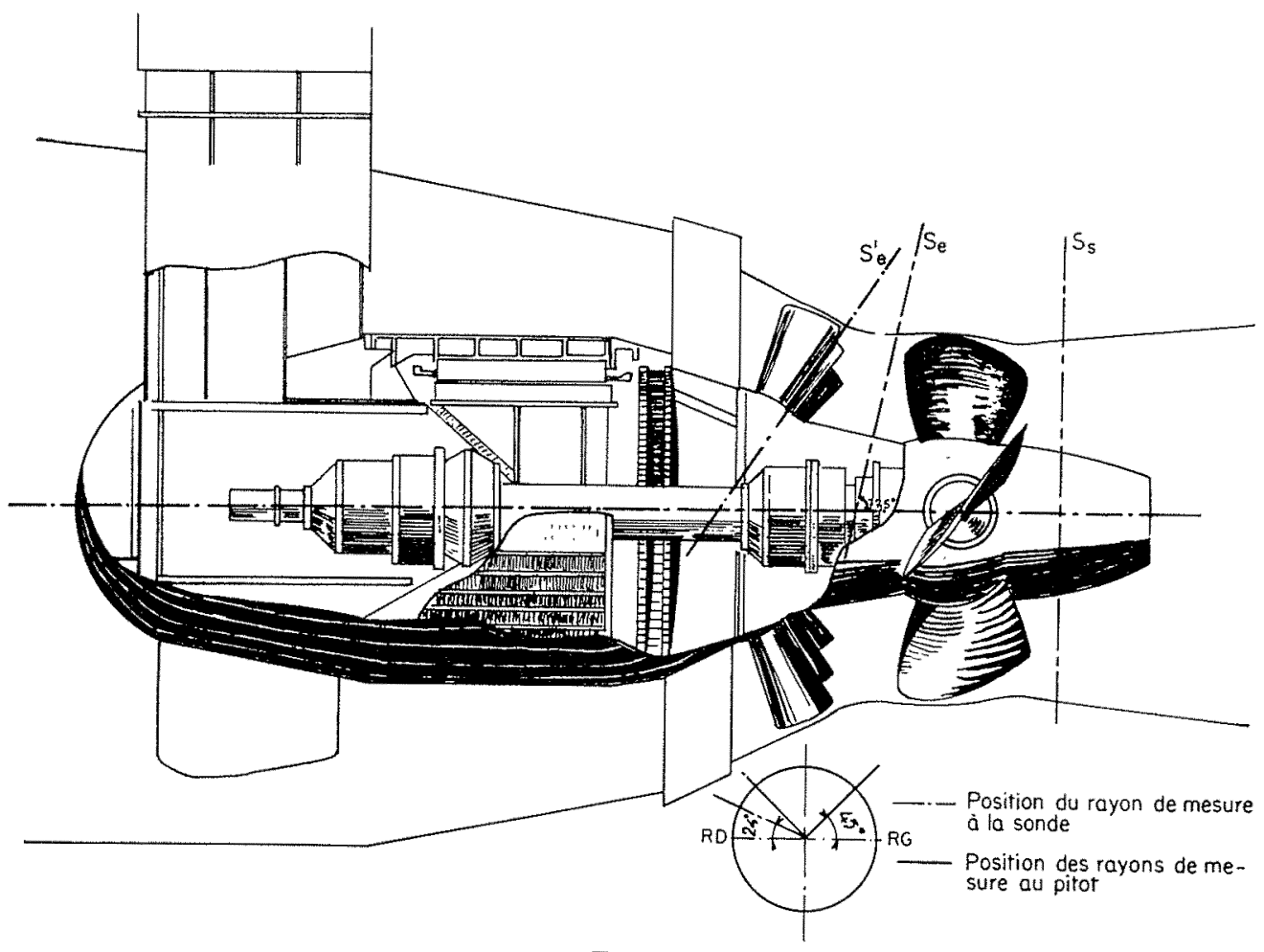

FIG. 1

Position des rayons de mesure et des sections de référence. 
grandeur et direction, en supposant toutefois la composante radiale négligeable (hypothèse vérifiée par l'expérience).

\section{Prospection de l'écoulement entre distributeur et roue.}

Elle a été exécutée suivant un seul rayon (repéré fig. 1) à peu près normal à la direction moyenne de l'écoulement. Pour permettre un effacement complet de l'appareil, la tête de mesure était constituée, non par un tube de Pitot, mais par une sonde spéciale à trois trous indiquant :

-.- directement la pression totale et l'orientation de la vitesse,

- moyennant un tarage préalable, la grandeur de la vitesse et la pression.

Ici encore on suppose que la composante de la vitesse suivant le rayon de mesure est négligeable.

\section{Repérage du régime de fonctionnement de la turbine.}

Il a été assuré par le relevé :

-_ de la position de pales et du vannage;
- de la hauteur de chute brute;

-_. de la puissance sur l'arbre.

\section{Nombre d'essais.}

Ont été effectuées :

15 prospections de l'écoulement entre distributeur et roue;

10 prospections de l'écoulement dans l'aspirateur suivant le rayon côté rive droite (qui sera noté $\mathrm{RD}$ );

57 prospections de l'écoulement dans l'aspirateur suivant le rayon rive gauche (RG), qui couvrent une large plage de part et d'autre de la conjugaison optimale pales-vannage (l'ouverture de ce dernier variant de $4 / 10$ à 10/10).

En raison du nombre élevé d'essais et de l'évolution assez régulière des caractéristiques de l'écoulement entre le manteau et le moyeu de la roue, chaque prospection n'a comporté que 10 points de mesure, ce qui correspond tout de même à un total de 820 relevés de pressions, vitesses et angles.

\section{RESULTATS}

Il ne saurait être question de reproduire ici les résultats détaillés de toutes les mesures effectuées; mais on dégagera progressivement les principales conclusions que l'on peut tirer de l'ensemble des essais.

En raison de son application pratique immédiate et évidente, on recherchera tout d'abord la conjugaison optimale entre pales et vannage en utilisant :

- soit les pertes en aval de la roue;

- - soit les rendements calculés à partir des dé- bits déduits des mesures au tube de Pitot dans l'aspirateur.

L'accord entre les deux procédés se révélera satisfaisant.

Le souci de confirmer la validité des rendements ci-dessus nous conduira à une étude des angles moyens d'entrée (application du théorème d'Euler) et de la symétrie de l'écoulement.

Enfin, connaissant le rendement, donc les pertes, nous tenterons de localiser ces dernières afin d'orienter d'éventuels efforts d'amélioration.

\section{I. - DETERMINATION DE LA CONJUGAISON OPTIMALE ENTRE PALES ET VANNAGE}

\section{I.1. - Recherche du minimum de pertes en aval de la roue.}

L'expérience montre qu'en général, si l'on déconjugue une turbine Kaplan, la plus grosse partie de la baisse de rendement provient d'une augmentation des pertes en aval de la roue. Aussi est-il possible de déterminer la conjugaison optimale avec une précision souvent suffisante dans la pratique en recherchant les points 


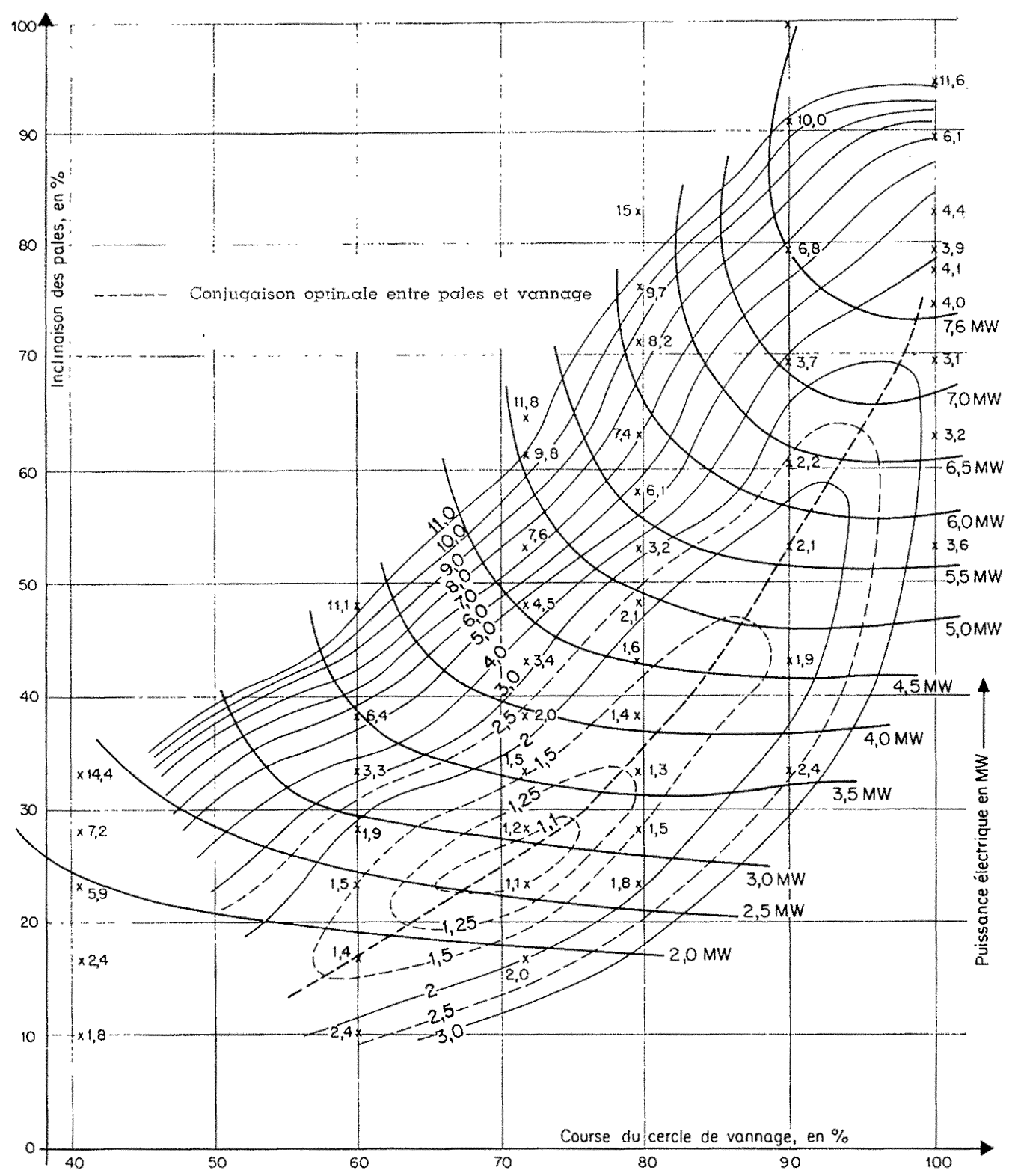

Fig. 2

Pertes en aval de la roue d'après les mesures sur le rayon RG.

de fonctionnement où, pour une puissance débitée donnée, les pertes en aval de la roue sont minimales.

Or ces dernières se déduisent immédiatement des mesures au tube de Pitot dans l'aspirateur. (en admettant que l'écoulement est de révolution autour de l'axe du groupe). Nous avons dressé leur colline à partir des résultats obtenus sur le rayon $\mathrm{RG}$ (fig. 2 ).

La conjugaison optimale s'obtient en joignant les zones médianes des segments découpés sur les courbes d'égales puissances par les lignes de niveau définissant le cour de la colline.

\section{I.2. - Utilisation du débit $\mathbf{Q}_{s}$ déduit des mesures au tube de Pitot dans l'aspira- teur.}

Malgré les bons résultats précédents, on peut regretter l'absence d'une vérification portant sur le rendement de la turbine. Or les seules mesures de débit que nous possédions sont les va- 


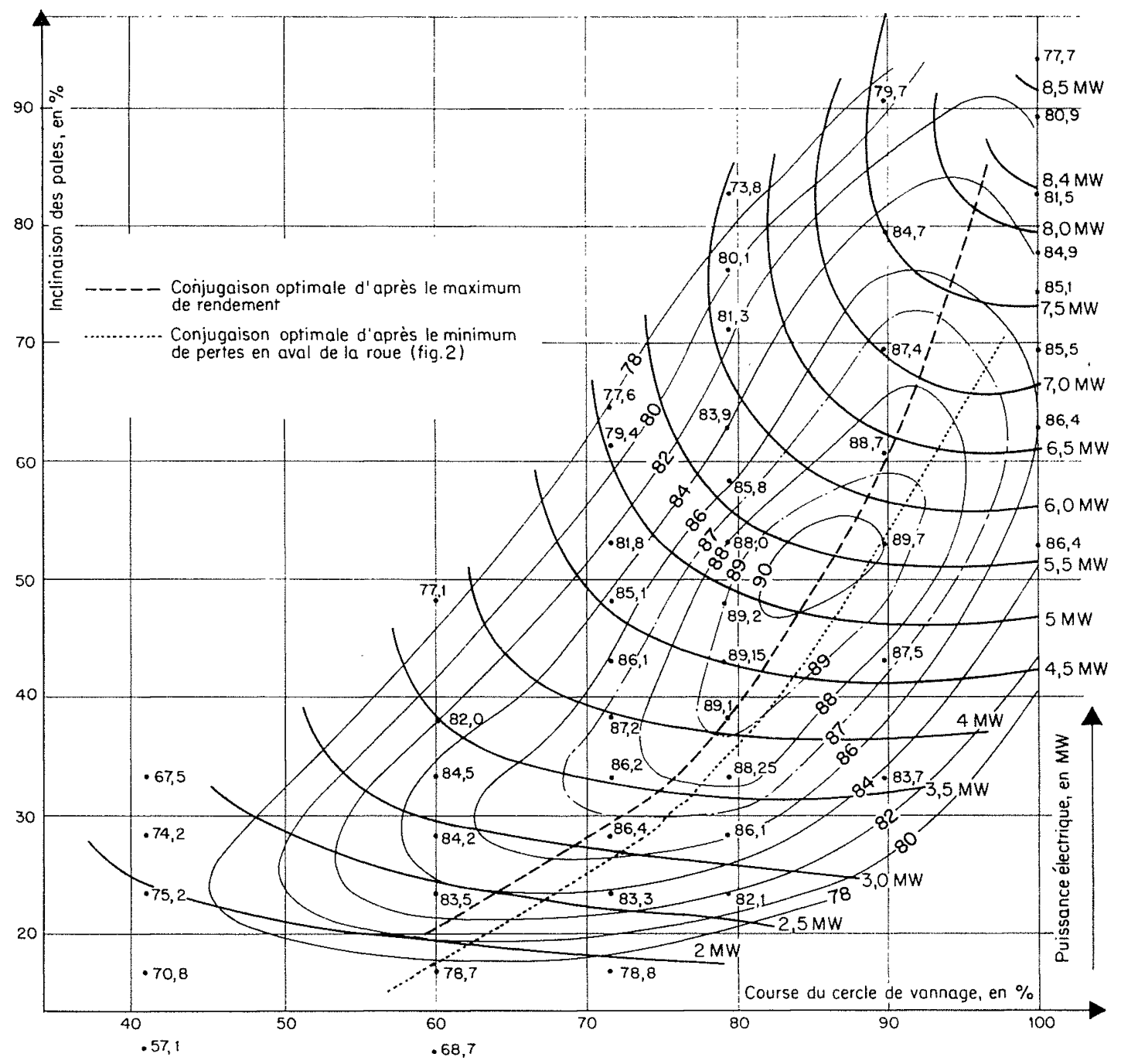

Fig. 3

Rendement de la turbine d'après les mesures sur le layon Ra.

leurs $Q_{s}$ déduites de la prospection de l'écoulement sous la roue avec le tube de Pitot.

Nous n'ignorons pas que le rendement ainsi calculé doit être utilisé avec une certaine prudence, mais il serait injustifié de renoncer a priori à une catégorie d'informations (2).

Les résultats obtenus sont représentés sur la figure 3 . On peut constater que les conjugaisons optimales définies sur les figures 2 et 3 ne coïncident pas exactement, mais que les écarts de

(2) Le calcul des pertes en aval de la roue utilise essentiellement les mesures de pression totale, le calcul du rendement celles de vitesse (dans l'aspirateur) et de puissance électrique. Ils font donc appel à des éléments assez différents. rendement entre les deux déterminations, en général de l'ordre de 0,1 à $0,3 \%$ et ne dépassant pas $0,5 \%$, ne sont guère significatifs. L'accord entre les deux méthodes (dont certains éléments sont rigoureusement indépendants) est donc satisfaisant. En outre, on n'observe aucune contradiction entre les variations des pertes totales dans la turbine et des pertes en aval de la rouc en fonction de la déconjugaison.

Le tracé de la conjugaison optimale peut donc être considéré comme acquis; mais ces concordances ne constituent pas une preuve suffisante de la validité des rendements calculés. L’application du théorème d'Euler entre l'entrée et la sortie de la roue nous fournit par contre une précieuse possibilité de vérification, 


\section{II. - VERIFICATION DES RENDEMENTS PAR APPLICATION DU THEOREME D'EULER ET ETUDE DES ANGLES MOYENS D'ENTREE}

L'application du théorème d'Euler dans les turbines est trop classique pour qu'il soit utile de rappeler l'expression du rendement à partir des ritesses à l'entrée et à la sortie de la roue.

Mais les résultats du calcul dépendent directement du tarage de la sonde utilisée en amont. Pour éliminer totalement ce paramètre, nous passerons par l'intermédiaire des angles moyens d'entrée dont la notion peut être introduite de la manière suivante.

Prenons comme surfaces de référence :

- à l'entrée de la roue le tronc de cône $\left(S_{e}\right)$ engendré par la rotation du rayon prospecté autour de l'axe du groupe;

- à la sortie, la section droite de l'aspirateux contenant les rayons de mesure.

$\rightarrow$

Soit Vle vecteur vitesse en un point $M$ de ces surfaces;

$d Q$ le débit massique à travers un élément de surface entourant le point $\mathrm{M}$;

$\mathrm{C}_{c}$ le couple, par rapport à l'axe, des vecteurs $\overrightarrow{\mathrm{V}} d \mathrm{Q}$ relatifs à $\left(\mathrm{S}_{\rho}\right)$;

$\mathrm{C}_{s}$ le même couple pour $\left(\mathrm{S}_{\mathrm{s}}\right)$.

Le théorème d'Euler nous apprend que le couple moteur est égal à $\mathrm{C}_{e}-\mathrm{C}_{s}$.

Considérons un écoulement fictif de même débit et de même couple $\mathrm{C}_{e}$ que l'écoulement réel et où la répartition des vitesses serait uniforme (3). Soit l'angle (constant) de la vitesse avec la normale à $\left(S_{e}\right)$.

Dans l'application du théorème d'Euler, les écoulements réels et fictifs sont rigoureusement équivalents, et, par convention, nous dirons que @ est l'angle moyen d'entrée.

Or, $\varphi$ se déduit immédiatement des seules mesures à l'entrée indépendamment du tarage de la sonde car, la grandeur de la vitesse n'intervenant que comme coefficient de pondération, $\vec{V}$ peut n'être connu qu'à un coefficient près (4). Nous désignerons les résultats ainsi obtenus par $\varphi_{0}$.

D'autre part, la prospection de l'écoulement dans l'aspirateur et la mesure de la puissance sur l'arbre nous donnent $\mathrm{C}_{s}$ et $\mathrm{Q}$, done $\mathrm{C}_{e}$ (théo-

(3) On suppose toujours que, comme dans l'écoulement réel, la composante méridienne de la vitesse est pratiquement perpendiculaire à $\left(\mathrm{S}_{e}\right)$.

(4) On trouvera en annexe l'expression mathématique de $\varphi$.

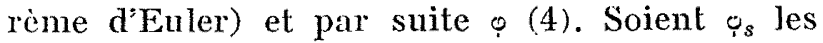
valeurs trouvées.

La comparaison de $\varphi_{s}$ et $\varphi_{e}$ va nous fournir une vérification des débits et des rendements déduits des mesures au tube de Pitot.

Examinons tout d'abord (iig. 4) les résultats relatifs à une position donnée du vannage (course $79 \%$ ).

On constate immédiatement que :

- les angles o sont, au moins en première approximation, indépendants de la position des pales;

- la différence entre les moyennes de $\varphi_{e}$ et $\varphi_{s}$ n'est que de 1,1 degré. En supposant que cet écart soit uniquement imputable à une erreur sur le débit Pitot $Q_{s}$, son incidence sur le rendement serait inférieure à deux points.

Or, il est normal que $q_{e}$, soit légèrement supé-
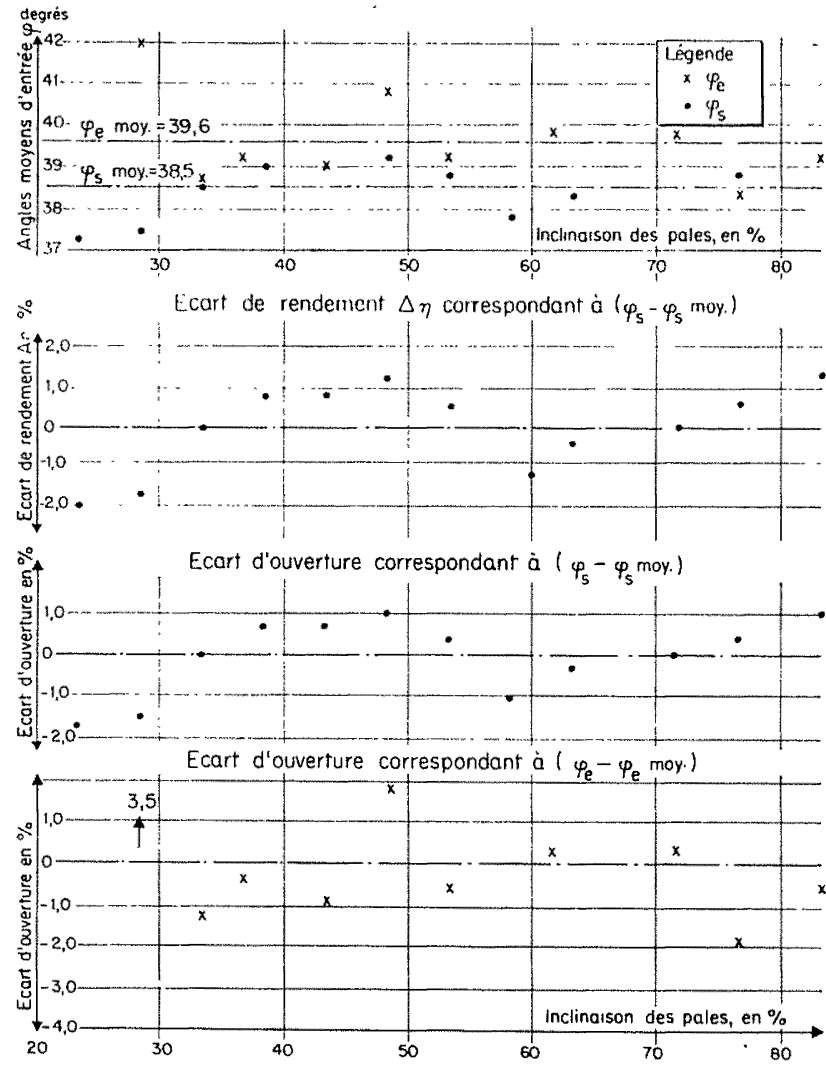

Fic. 4

Etude des angles moyens d'entrée pour $79 \%$ d'ouverture du distributeur. 
rieur à is: en effet, pour appliquer le théorème d'Euler , nous aurions dû tenir compte des pertes mécaniques et, dans une certaine mesure, des pertes marginales (entre pales et manteau) (5). Nous ignorons l'importance de ces termes correctifs, mais nous savons qu'ils conduiraient à une augmentation du rendement utilisé, donc de $\varphi_{s}$.

Le recoupement entre les deux minéthodes est donc tout à fait satisfaisant; il montre l'absence d'erreur systématique dans les mesures au tube de Pitot.

Remarquons que la constance de $\varphi_{e}$ et $\varphi_{\beta}$ est particulièrement marquée pour une ouverture des pales supérieure à $33 \%$ : dans cette zone, l'écart moyen n'est que de 0,65 degré (soit 1 à 1,2 points de rendement).

Reste à examiner la dispersion des angles autour de leur valeur moyenne : traduite en points de rendement (6), elle reste pour ?s toujours inférieure à deux points. De plus, nous n'avons pu obtenir une stabilité parfaite de la position du vannage, dont l'influence est faible ou même pratiquement nulle sur le rendement, mais fort importante sur is (et io). C'est pourquoi les irrégularités constatées ne sont pas significatives et on peut considérer que cet angle est indépendant de la position des pales (i).

(5) Voir annexe.

(6) En supposant toujours qu'elle est impulable en lotalité à une erreur sur le débit.

(7) Il paraît tout à fait justifié d'admettre que $\varphi$ est également indépendant des légères variations de hauteur de chute existant entre certains essais. Nous n'aurions pu les négliger dans une application directe du théorème d'Euler.
Il en est de même pour $\varphi_{e}$, la mesure expérimentale précise de cet angle présentant quelques difficultés.

Cette conclusion suppose un excellent guidage de l'eau par les directrices dans un large domaine entourant la conjugaison optimale. S'il en est ainsi, on doit retrouver cette propriété aux autres ouvertures du distributeur.

C'est effectivement ce que l'on constate sur la figure 5 (8).

Dès lors, une comparaison avec la position des directrices s'impose. Pour être parfaitement valable, cette comparaison devra se faire immédiatement à la sortie du distributeur, par exemple dans la section conique de révolution $\left(S_{o}^{\prime}\right)$ tangente à la pointe des directrices, où nous pourrons définir des angles moyens $\varphi^{\prime}$ :légèrement différents de $\varphi$, car les caractéristiques de $\left(\mathrm{S}_{e}\right)$ et de $\left(\mathrm{S}_{e}^{\prime}\right)$ ne sont pas identiques :

$$
\left.\operatorname{tg} \varphi^{\prime}=0,977 \operatorname{tg} \varphi(1)\right]
$$

En fait, pour simplifier les calculs, nous nous sommes contentés de ramener fictivement dans la section $\left(S_{e}\right)$ (par la relation (1) ci-dessus) l'angle $\varphi_{d}^{\prime}$ de l'axe de la pointe des directrices $(9)$; $\varphi_{d}$ et la moyenne de $\varphi_{s}$ coincident à mieux que 1 degré près (10).

(8) Sauf peut-ĉtre à partir d'une certaine déconjugaison côté fermeture de la roue, mais les anomalies constatées dans cette zone ne sont peut-être pas significatives.

(9) $\varphi^{\prime} d$ a été directement mesuré au cours des essais.

(10) Nous attachons plus d'importance à l'égalité des variations de $\varphi_{a}$ et $\varphi$ qu'à la coüncidence de leurs valeur's absolues. En effet, $\Delta_{\varphi_{d}}$ (directement et aisément mesurable) est a priori connu avec plus de précision que $\varphi^{d}$ (incertitude sur la définition et le repérage de l'axe de la pointe des directrices).

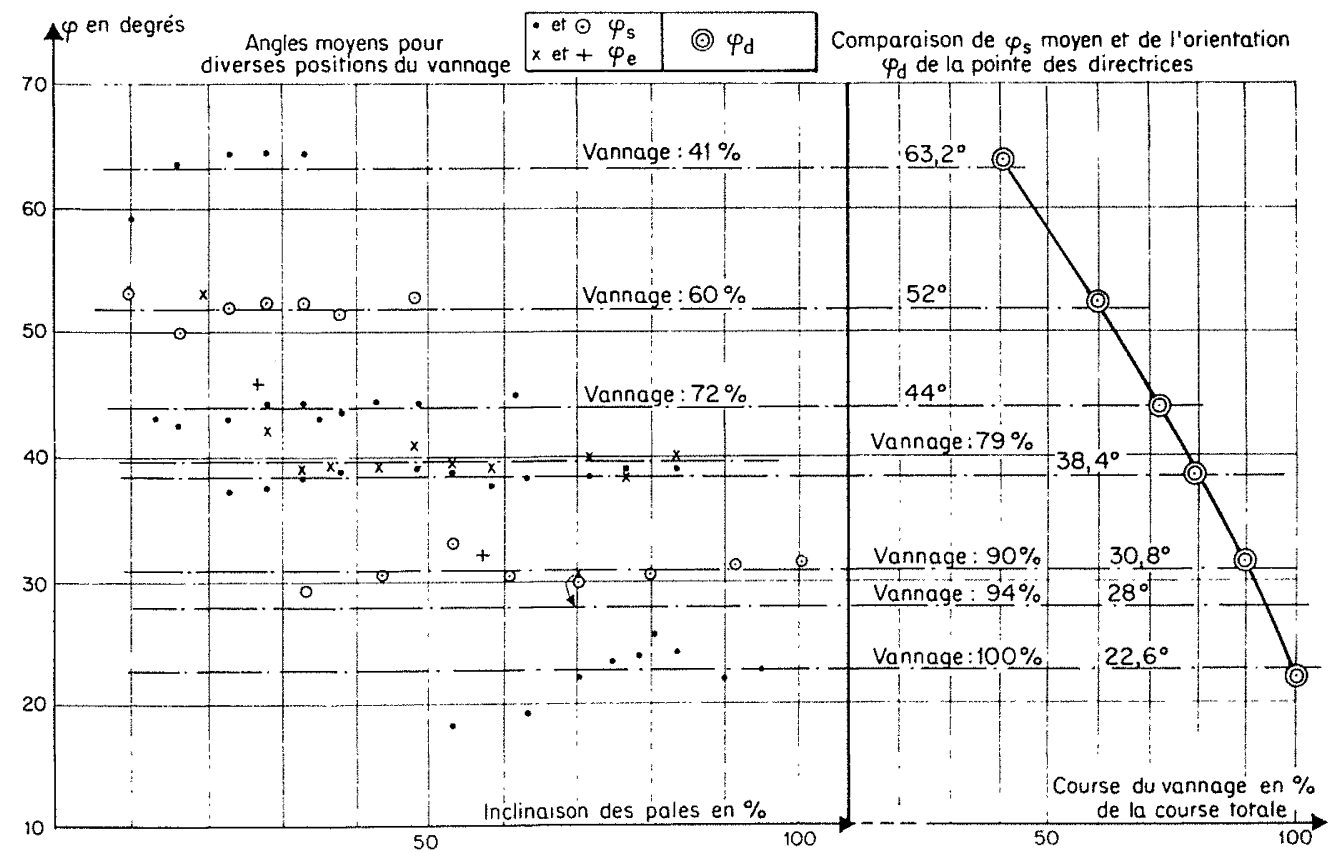

FIG. 5

Comparaison des angles moyens d'entrée et des rotations des directrices. 
Quant aux valeurs de of, elles sont trop peu nombreuses pour permettre une étude approfondie. Bornons-nous à constater qu'elles se plasent à 1,6 degré en moyenne au-dessus des paliers de $\varphi_{s}$.

En conclusion, compte tenu de la remarquable cohérence des diverses mesures indépendanles effectuées, on ne peut mettre en évidence aucune erreur systématique importante (dépassant 2 points pour fixer les idées) entachant l'ensemble des calculs du rendement à partir des indications du tube de Pitot.

\section{III. - ETUDE DE LA SYMETTRIE DE L'ÉCOULEMENT}

Dans lout ce qui prècède, nous n'avons utilisé que les mesures dans l'aspirateur exécutées sur le rayon $R G$ et nous avons admis que l'écoulement était de révolution autour de l'axe du groupe. Rien dans les résultats obtenus ne laisse supposer qu'il n'en est pas ainsi. Toutefois, nous avons recherché une confirmation directe en réalisant quelques explorations de contrôle (au nombre de 10) sur le rayon $R D$.

La comparaison des deux rayons présente d'ailleurs quelques difficultés dues aux petites variations de hauteur de chute que nous n'avons pu éviter entre les deux séries d'essais : nous devrons appliquer aux principales caractéristiques de l'écoulement, que nous allons examiner successivement, de légères corrections en utilisant les courbes en colline du modèle réduit (11).

\section{III.1. - Pertes en aval de la roue.}

Pour une position $y$ du vannage, une variation $\Delta H_{b}$ de la hauteur de chute fait varier la position optimale des pales d'une quantité $\Delta i$ (fonction de $\Delta \mathrm{H}_{b}$ et $y$ ).

En l'absence de dissymétrie de l'écoulement, les courbes en vannage bloqué tracées en fonction de $i-\Delta i$ doivent donc définir la même conjugaison optimale.

Adoptant pour $\Delta i / \Delta H_{b}$ les valeurs déterminées sur modèle réduit, nous avons ramené toutes les positions des pales à une même chute moyenne et nous avons pu dresser le tableau ci-contre.

Si l'on excepte le dernier point (vannage $100 \%$ sur lequel nous reviendrons), on ne peut considérer ces différences conme significatives $(0,3 \%$ correspondent à $0,03 \mathrm{~m}$ (le chute).

(11) In analyse relativement complète ne pouvait étre envisagée en l'absence d'information sur le fonctionnement de la turbine. C'est pourquoi nous n'avons pu placer ce chapitre en tête du rapport (position a priori la plus logique).

Les variations de chute ont également affecté certains des résultats antérieurs mais elles ne se traduisent que par une légère dispersion des points sans altération des valeurs moyennes, donc des conséquences que nous en avons tirées.

\begin{tabular}{|c|c|c|}
\hline $\begin{array}{c}\text { Vannage } \\
\%\end{array}$ & $\begin{array}{c}\text { Pales } \\
\text { (sous chute } \\
\text { d'essai) } \\
\%\end{array}$ & $\begin{array}{c}\text { Pertes } R D \\
\text { - pertes } R G \\
\% \text { de } \mathrm{H}_{3}\end{array}$ \\
\hline 60 & 28 & $+0,2$ \\
\hline 72 & 38 & $+0,3$ \\
\hline \multirow{5}{*}{79} & 28 & $+0,1$ \\
\hline & 33 & $+0,1$ \\
\hline & 38 & $-0,2$ \\
\hline & 48 & $-0,2$ \\
\hline & 58 & $-0,2$ \\
\hline 90 & 61 & $+0,1$ \\
\hline 100 & 75 & $+1,3$ \\
\hline
\end{tabular}

A titre de vérification de la méthode, nous disposons de cinq points de contrôle sur le rayon RG dont l'écart de chute par rapport à la série principale est supérieur à celui de mesures RD.

\begin{tabular}{|c|c|c|}
\hline $\begin{array}{c}\text { Vannage } \\
\%\end{array}$ & $\begin{array}{c}\text { Pales } \\
\text { (sous chute } \\
\text { d'essai) } \\
\%\end{array}$ & $\begin{array}{l}\text { Erreur } \\
t o \text { de } \mathrm{H}_{b}\end{array}$ \\
\hline 60 & 20 & $0,2(1)$ \\
\hline 72 & 28 & $<0,1(1)$ \\
\hline \multirow{2}{*}{79} & 37 & $0,1(1)$ \\
\hline & 48 & $\begin{array}{r}<0,1(2) \\
0,4(3)\end{array}$ \\
\hline 90 & 58 & $<0,1(1)$ \\
\hline \multicolumn{3}{|c|}{$\begin{array}{l}\text { Observation: } \\
\text { (1) Pratiquement indépendante de la correc- } \\
\text { tion de chute. } \\
\text { (2) Avec correclion de chute. } \\
\text { (3) Sans correction de chute. }\end{array}$} \\
\hline
\end{tabular}


Après correction de chute, l'accord est fort satisfaisant.

En conclusion, toutes choses restant égales par ailleurs, les pertes en aval de la roue (et la conjugaison optimale entre pales et vannage) déduites des deux rayons $R D$ et $R G$ sont praiiquement confondues dans le domaine exploré.

\section{Remarque.}

On aurait pu appliquer les corrections ci-dessus à l'ensemble des mesures RG : la colline de la figure 2 aurait été plus « régulière 》. Mais nous avons préféré, pour un tel élément de base, n'utiliser que nos propres résultats d'essai.

\section{III.2. - Débit $\mathrm{Q}_{s}$, rendement $\eta$ et angle moyen d'entrée @.}

Il est difficile de séparer trois éléments aussi étroitement liés. En utilisant comme précédemment les caractéristiques du modèle réduit, il est très facile de ramener les débits à même hauteur de chute (celle des essais RD).

Dans les expressions de $r_{\text {}}$ et $\varphi$ intervient la puissance sur l'arbre dont nous ne possédons qu'un contrôle sommaire au cours des prospections RD. Aussi admettrons-nous que les différences relatives de $Q_{s}$ et $\eta$ entre les deux rayons sont égales et de signes opposés (12).

On en déduit le rendement RD (13), les angles moyens d'entrée et finalement le tableau comparatif suivant.

\begin{tabular}{|c|c|c|c|c|c|c|}
\hline & $\begin{array}{c}\text { Vannage } \\
o n\end{array}$ & $\begin{array}{l}\text { Pales } \\
\%\end{array}$ & $\frac{Q_{\mathrm{ND}}-Q_{\mathrm{RG}}}{Q_{\mathrm{RG}}}$ & $\begin{array}{c}\eta_{R D}-\eta_{\mathrm{KG}} \\
\text { points }\end{array}$ & $\begin{array}{c}\left(\varphi_{s}\right)_{\mathrm{RD}}-\left(\varphi_{s} \text { moy }\right)_{\mathrm{Rt}} \\
\text { degrés }\end{array}$ & $\begin{array}{c}\left(\varphi_{\mathrm{s}}\right)_{\mathrm{nt}}-\left(\varphi_{s} \text { mos }\right)_{\mathrm{ro}} \\
\text { (pour mémoire }) \\
\text { dlegrés }\end{array}$ \\
\hline $1 \ldots \ldots$ & 60 & 28 & 1,8 & $-1,5$ & $-0,4$ & 0,3 \\
\hline $2 \ldots \ldots \ldots$ & 72 & 38 & -1 & 0,9 & 0,2 & $-0,1$ \\
\hline $3 \ldots \ldots \ldots$ & \multirow{5}{*}{79} & 28 & $-3,6$ & 3,1 & 1,7 & $-1,0$ \\
\hline $4 \ldots \ldots \ldots$ & & 33 & $-2,2$ & 1,9 & 1,2 & 0 \\
\hline $5 \ldots \ldots \ldots$ & & 38 & $-0,5$ & 0,4 & $-0,4$ & 0,5 \\
\hline $6 \ldots \ldots \ldots$ & & 48 & $-1,6$ & 1,4 & 0,4 & 0,7 \\
\hline $7 \ldots \ldots \ldots$ & & 58 & $-0,7$ & 0,6 & $-0,6$ & $-0,7$ \\
\hline $8 \ldots \ldots$ & 90 & 61 & $\cdots-2,2$ & 2,0 & $-0,8$ & $\ldots 0,4$ \\
\hline$\ldots \ldots$ & 100 & 75 & $\ldots 0,7$ & 0,6 & $-\cdots 3,7$ & 0,0 \\
\hline
\end{tabular}

Mis à part $\left(\varphi_{s}\right)_{\mathrm{RD}}$ à $100 \%$ d'ouverture du vannage, on voit que, dans l'ensemble, ces différences, pour $Q$ et $\eta_{1}$ calculées directement entre points correspondants considérés isolément, sont peu importantes.

Le seul écart vraiment notable sur $Q$ et $\eta$ apparaît au point 3 , vraisemblablement parce qu'il y a eu à la fois surestimation de $\eta_{\mathrm{RD}}$ et sousestimation de $\eta_{\mathrm{RG}}$.

Mais, d'une manière générale, il est difficile de faire la part des inévitables erreurs de me-

(12) Ce qui est d'ailleurs parfaitement logique dans une étude ne portant que sur la comparaison des rayons $\mathrm{RD}$ et $\mathrm{RG}$.

(13) Ramené à la chute d'essai de la série RG. Sous une chute différente, on peut admettre que

$$
\eta \mathrm{RD}=\eta_{\mathrm{RG}}+\varepsilon_{1}+\varepsilon_{\mathrm{q}}
$$

$\varepsilon_{1}$ incidence de l'écart de chute sur le rendement du modèle réduit,

E2 influence de la dissymétrie en débits (tableau cidessus). sure et d'une dissymétrie réelle de l'écoulement. Dans le cadre des tolérances que nous nous sommes fixées au chapitre précédent $(2$ points de rendement environ), les deux rayons peuvent être considérés comme identiques.

Avant de terminer ce paragraphe, évoquons les deux problèmes qui subsistent :

- A pleine charge, il existe une singularité entre rayons $R D$ et $R G$. Nous ignorons s'il faut attribuer ce fait à un phénomène physique ou à une erreur de mesure (14). Cette incertitude n'a aucune conséquence pratique : dans cette région, la conjugaison optimale commence à être mal définie et la position des pales sera essentiellement fixée en fonction de la puissance recherchée.

(14) En particulier, aux fortes charges, les risques de dégazage dans la partie invisible des circuits de mesure sont plus grands. 
- Existe-t-il une dissymétrie plus importante en des points non prospectés? Une certitude absolue est évidemment impossible, mais la cohérence des résultats laisse supposer qu'il n'en est rien. Un nouvel argument nous est d'ailleurs fourni par le tarage de la sonde amont.

\section{III.3. - Tarage en vitesse de la sonde utilisée à l'amont.}

Nous avons exécuté un tarage dans les conditions mêmes d'utilisation en comparant six mesures simultanées de débit avec la sonde et le tube de Pitot placé sur le rayon RG de l'aspirateur.

Le tableau ci-contre indique l'écart relatif entre la constante de tarage $k$ résultant de chaque essai et sa vąleur moyenne $k_{m}$ sur les six essais.

On voit qu'il n'apparait aucune singularité significative en fonction de la charge, ce qui sup. pose soit une assez bonne symétrie de l'écoulement, soit, hypothèse plus difficile à admettre, la conservation, en valeur relative, de l'anomalie de répartition des vitesses qui pourrait éventuellement exister dans une zone non étudiée.

\begin{tabular}{|c|c|c|}
\hline $\begin{array}{c}\text { Vannage } \\
\%\end{array}$ & $\begin{array}{c}\text { Pales } \\
\%\end{array}$ & $\frac{k-k_{m}}{\begin{array}{c}k_{m i} \\
\%\end{array}}$ \\
\hline 60 & 20 & 1,2 \\
\hline 72 & 28 & 0 \\
\hline 79 & 37 & $-1,3$ \\
\hline 79 & 48 & $-0,9$ \\
\hline 90 & 58 & 0,6 \\
\hline 94 & 68 & 0,5 \\
\hline
\end{tabular}

\section{IV. - LOCALISATION DES PERTES DANS LA TURBINE}

L'un des principaux buts du chapitre précédent était la justification des figures 2 (pertes en aval de la roue) et 3 (rendement, donc pertes totales) sur lesquelles nous lirons désormais les valeurs numériques que nous utiliserons.

Mais le calcul de l'altitude moyenne de la ligne de charge dans la section $\left(S_{e}\right)$ (indépendante du tarage en vitesse de la sonde) nous fournit également la valeur des pertes en amont de la roue, donc, par différence, dans la roue proprement dite.

Le résultat de cette ventilation des pertes entre les divers organes de la machine est indiqué sur la figure 6 pour un fonctionnement en conjugaison (15).

On notera la faible valeur des pertes en aval de la roue qui croissent d'ailleurs moins vite que le carré du débit : l'amélioration que nous avons pu constater dans la répartition des vites-

(15) C'est-à-dire pour la conjugaison optimale entre pales et vannage. Nous avons adopté la position moyenne entre le minimum des pertes en aval de la roue et le maximum du rendement déduit du Pitot.

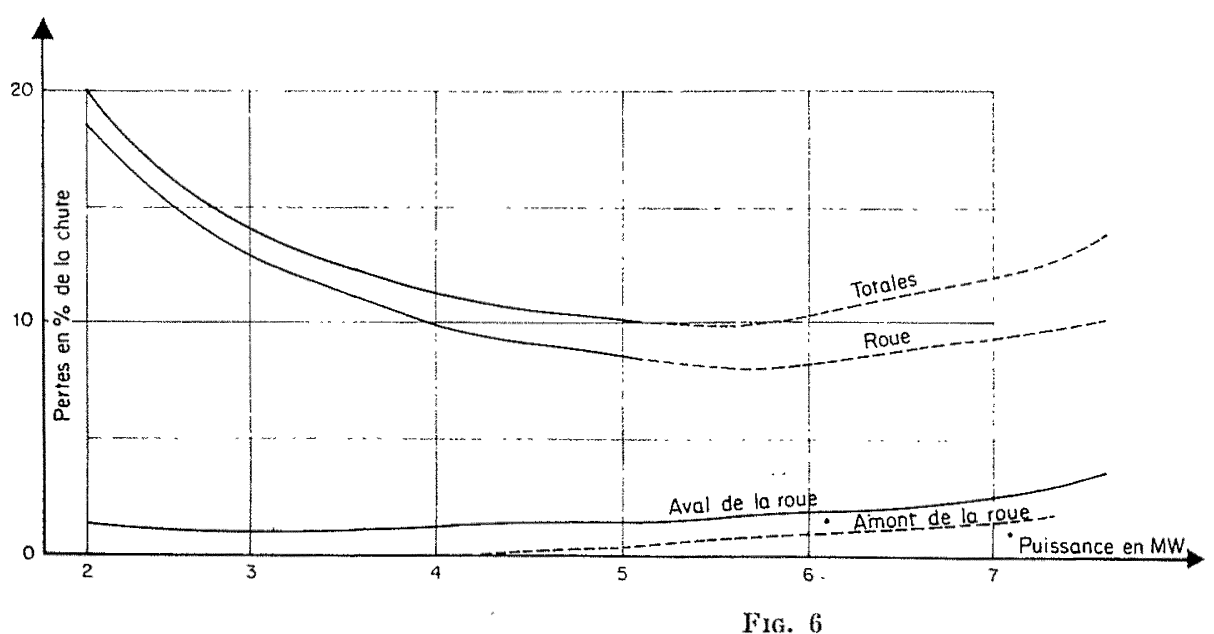

Ventilation des pertes entre les divers organes de la turbine. 
ses et des angles explique vraisemblablement l'augmentation, très souhaitable d'ailleurs, du coefficient de récupération de l'aspirateur aux fortes charges, qui atteint des valeurs de l'ordre de $90 \%$.

Les pertes mesurées comprennent l'énergie cinétique obligatoirement perdue à la sortie de l'aspirateur, soit $1 \%$ à $7 \mathrm{MW}$ sur une perte totale de $2,8 \%$. On voit que des gains appréciables dans celte partie de la machine sont pratiquement impossibles.

Les pertes en amont de la roue ne sont pas mesurables au-dessous de $4 \mathrm{MW}$. Au-delà, nous ne disposons que de deux points un peu dispersés, mais montrant qu'elles restent également très faibles.

La roue proprement dite est donc le siège des pertes les plus élevées et c'est sur elle que doivent porter les éventuels efforts d'amélioration.

\section{V. - INDICATIONS SUR LE DÉTAIL DE L'ECOULEMENT}

Après cette longue étude des caractéristiques globales de l'écoulement, donnons quelques indications sur sa forme exacte.

Une vue d'ensemble est fournie par la figure 7, qui indique la répartition des vitesses et des angles en aval et en amont de la roue pour quelques marches en conjugaison (conditions normales de fonctionnement).

Pour suivre plus facilement l'évolution des caractéristiques entre sections différentes, il est commode d'introduire le débit relatif $q$ défini sur la figure 8 : dans un écoulement de révolution, les points correspondant à une inême valeur de $q$ sont relatifs à des filets liquides identiques et sont donc directement comparables.

Pour l'entrée de la roue, il est particulièrement instructif d'étudier en fonction de $q$ le couple $e$ du vecteur vitesse de l'eau par rapport à l'axe de la turbine. Rapporté à sa valeur moyenne $e_{m}$ dans la section, il apparaît comme indépendant de la position des pales : à $79 \%$ d'ouverture du distributeur, nous n'avons pu mettre en évidence une modification de la loi $e / e_{m}=f(q)$ quand les pales passent de 28 à $83 \%$ d'ouverture (16).

$\mathrm{Si}$, de plus (figure 9), on compare cette loi expérimentale à celle, théorique, d'un écoulement :

- à vitesses uniformes dans le distributeur;

- sans échange de quantité de mouvement entre filets liquides voisins,

on trouve une similitude assez satisfaisante dans l'ensemble, à l'exception du point singulier au voisinage du manteau (diminution systématique de la vitesse). A titre indicatif, nous mentionnons la courbe que l'on obtiendrait en faisant abstraction de ce point (dans l'hypothèse d'une erreur de mesure ou d'un défaut de symétrie).

Quoi qu'il en soit, les vitesses dans le dis-

(16) Nous disposons au total de 10 prospections dans cette plage. tributeur ne s'écarteraient guère d'une répartition uniforme dans la plus grande partie ou même la totalité du distributeur.

Remarquons que, même dans l'hypothèse d'une erreur sur le point voisin du manteau, le calcul de l'angle moyen d'entrée n'est pratiquement pas affecté; en effet, $\alpha$ ne varie que de quelques degrés le long du rayon de mesure : il faudrait une altération très importante de la vitesse (qui n'intervient que comme coefficient de pondération) pour modifier $\varphi_{e}$ de façon non négligeable.

$\mathrm{Si}$, maintenant, on compare les écoulements à l'entrée et à la sortie de la roue, on peut, du

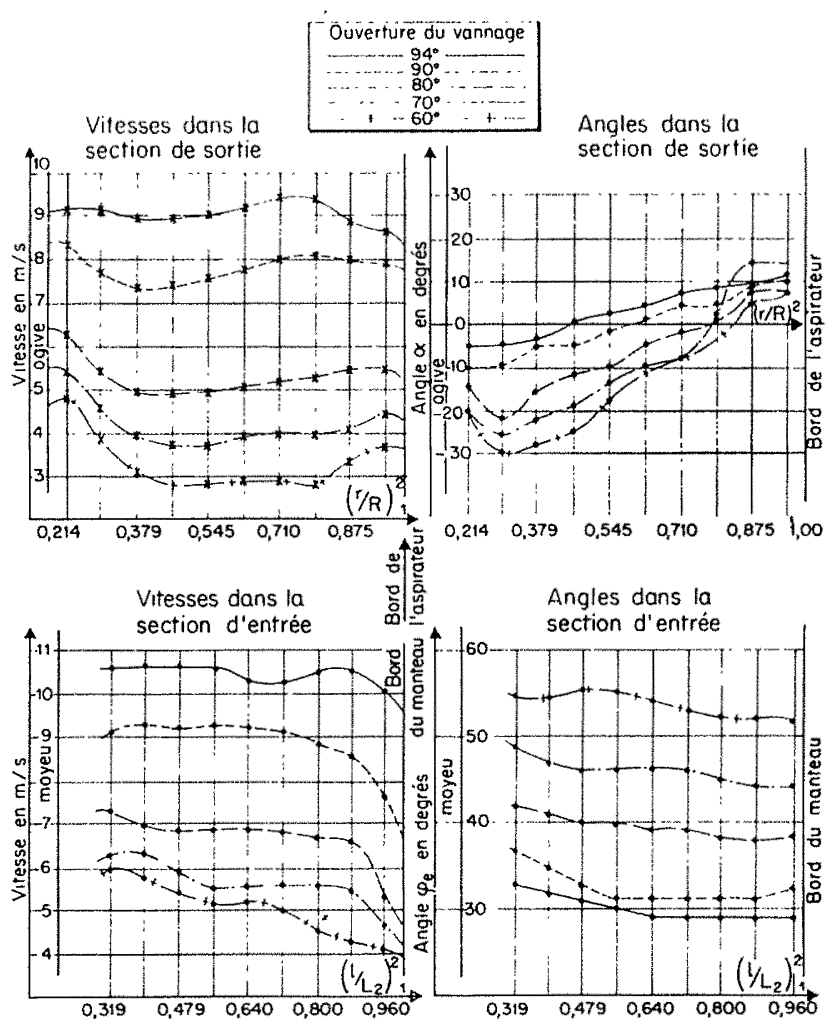

Frg. 7

Exemple de répartition

de vitesses et d'angles à l'entrée et à la sortie de la turbine (marche en conjugaison). 

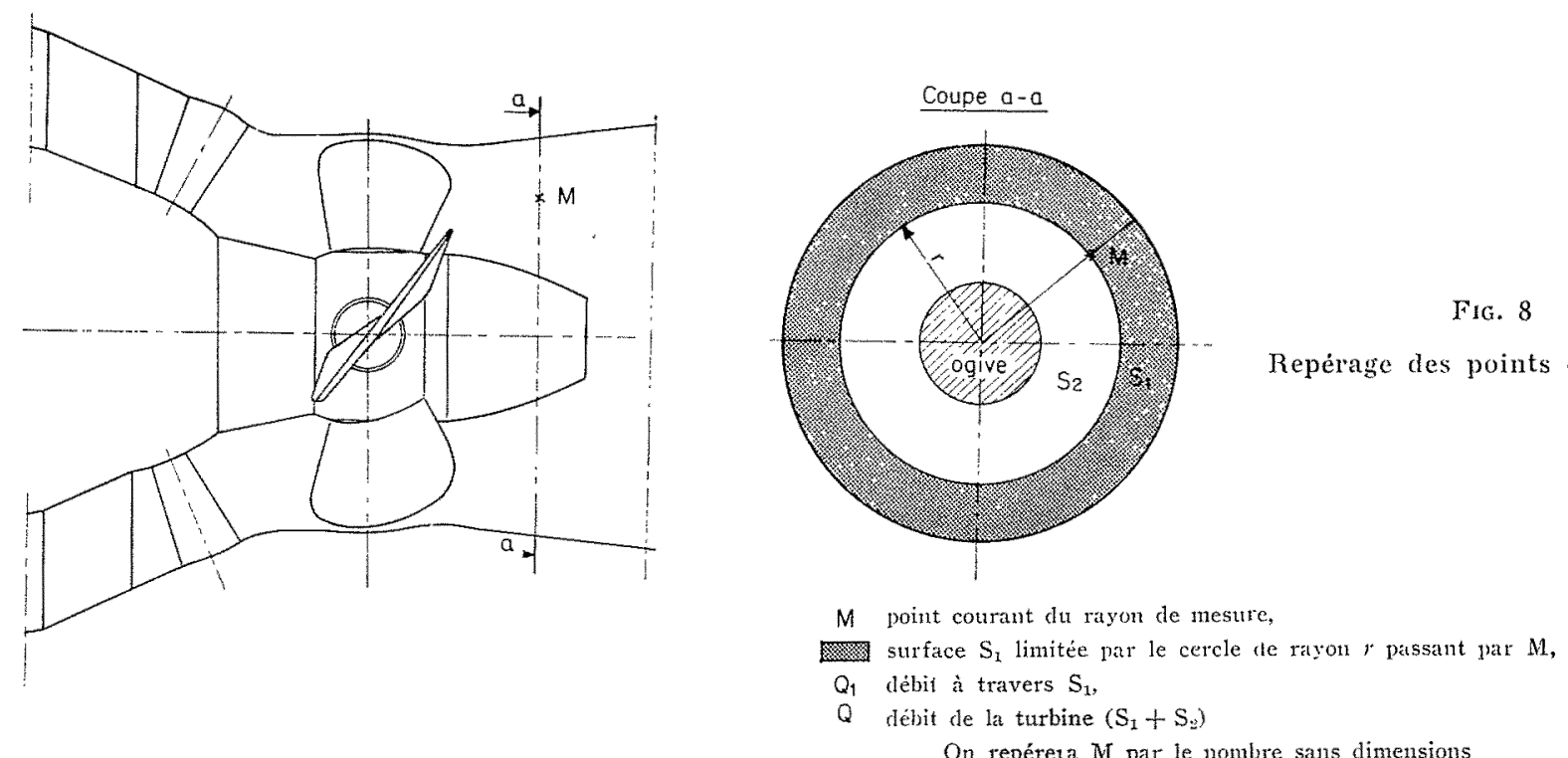

Fra. 9

Entrée de la roue. Variation du couple $e$ du vecteur vitesse en fonction de $q$.
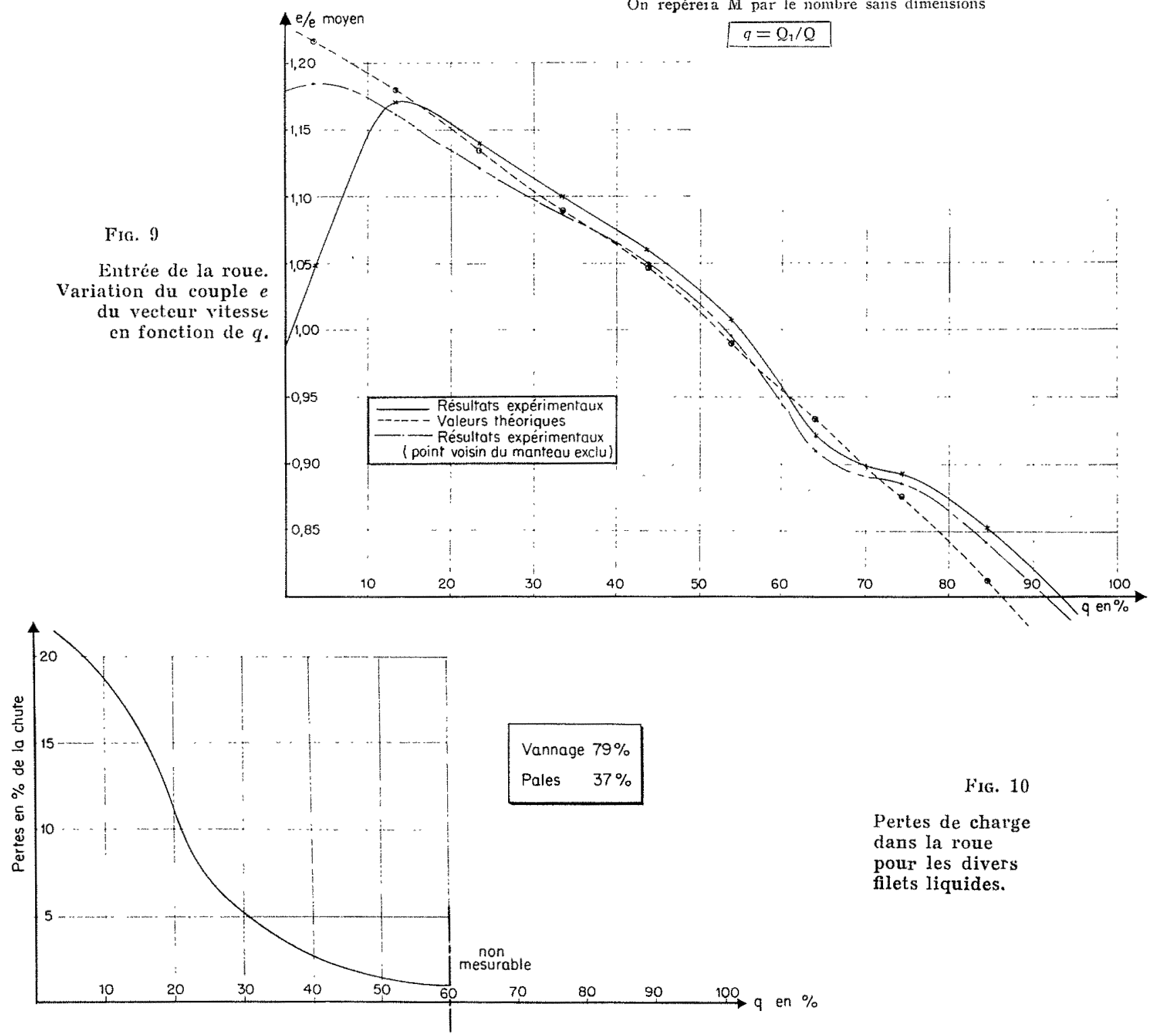

Fig. 10

Pertes de charge dans la roue pour les divers filets liquides. 
moins théoriquement, évaluer les pertes de charge pour les divers filets liquides (sous réserve qu'il n'y ait pas d'échanges d'énertie entre eux). A titre indicatif, nous avons effectué cette détermination à $79 \%$ d'ouverture du distributeur et $37 \%$ d'ouverture des pales (conjugaison optimale); on voit sur la figure 10 qu'il existerait une différence notable de comportement suivant la zone de pale intéressée. Malgré tout l'intérêt de ce résultat, nous n'insisterons pas davantage, car nous atteignons la limite de la signification des mesures.

\section{CONCLUSION}

L'exécution et le dépouillement des mesures que nous venons d'analyser représente un travail considérable. Mais, à l'origine, les essais n'avaient que des buts assez modestes; leurs objectifs ont évolué progressivement en fonction des résultats obtenus. C'est ce qui explique que dans l'optique finale du présent rapport, ils comportent des répétitions et aussi des lacunes. Une nouvelle expérimentation sur une turbine semblable serait à la fois plus complète et plus légère.

En montrant qu'il est possible dans certains cas d'obtenir le débit avec une précision comparable à celle des procédés classiques, ces essais marquent une étape importante dans l'évolution des mesures au tube de Pitot. Il ne s'agit pas là d'un résultat isolé : un essai récent conduit à un recoupement tout à fait satisfaisant avec des mesures aux moulinets.

Or, la méthode par le tube de Pitot ne conduit pas à une simple constatation de la valeur du rendement; elle fournit un certain nombre d'indications sur le comportement de la turbine, dont la valeur peut être considérablement augmentée si l'on peut accéder à l'amont de la rouc.

Evidemment, de nouvelles comparaisons sont encore indispensables; tous les problèmes relatifs à l'expérimentation et à l'interprétation des mesures ne sont pas résolus. Mais les résultats déjà acquis justifient dès maintènant :

- sur les grosses installations en construction, l'aménagement des accès nécessaires à l'exécution d'essais dans des conditions satisfaisantes;

-- sur une ou plusieurs machines présentant les dégagements nécessaires, le renouvellement de mesures complètes destinées à poursuivre l'analyse de l'écoulement dans les turbines Kaplan en vue d'obtenir le meilleur fonctionnement possible. 


\author{
ANNEXE \\ UTILISATION DU THEOREME D'EULER \\ POUR CARACTERISER LA ROTATION DE L'EAU \\ ET CALCULER DES ANGLES MOYENS D'ENTREE
}

\title{
I. - PRINCIPALES NOTATIONS
}

Soit :

W la puissance sur l'arbre de la turbine;

$\mathrm{H}_{n}$ la hauteur de chute nette;

$\mathrm{N}$ la vitesse de rotation;

क le poids spécifique de l'eau;

$g$ l'accélération de la pesanteur;

$\eta$ le rendement de la turbine.

Nous considérerons deux surfaces de référence
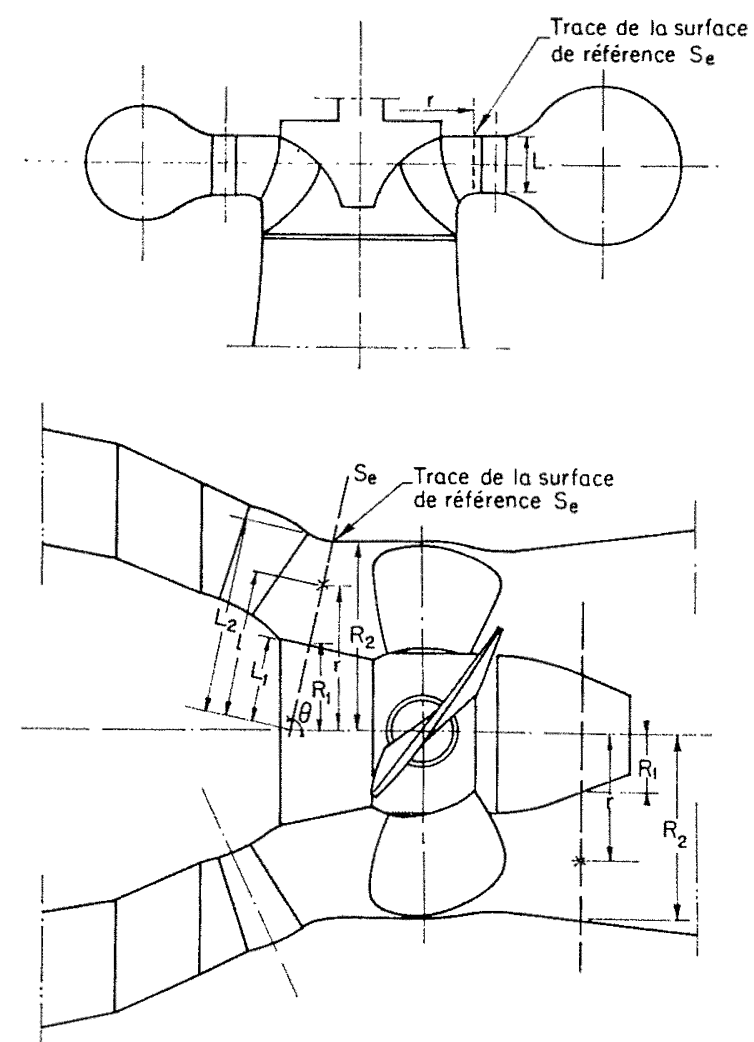

Fig. 11

Calcul des angles moyens d'entrée.
$\left(\mathrm{S}_{e}\right)$ et $\left(\mathrm{S}_{s}\right)$ respectivement en amont et en aval de la roue, de révolution autour de l'axe de la turbine et sensiblement normales à l'écoulement méridien. En pratique, $\left(\mathrm{S}_{s}\right)$ sera toujours une section plane de l'aspirateur, $\left(\mathrm{S}_{e}\right)$ une surface plane, conique ou eylindrique.

Dans ces sections nous désignerons par (fig. 11) :

$\mathrm{V}$ la vitesse de l'eau en un point donné M;

\% l'angle de la vitesse avec la normale à la surface $(\mathrm{S})$ en $\mathrm{M}$;

$l$ l'abscisse de $\mathbf{M}$ mesurée sur la génératrice correspondante (origine : l'axe du groupe ou, dans le cas d'un cylindre, l'une des bases);

$\mathrm{L}_{1}$ et $\mathrm{L}_{2}$ les valeurs extrêmes de $l$;

L la quantité $\mathrm{L}_{2}-\mathrm{L}_{1}$;

$r$ distance de $\mathrm{M}$ à l'axe du groupe;

$R_{1}$ et $R_{2}$ valeurs extrêmes de $r$;

$\mathrm{R}$ la quantité $\mathrm{R}_{2}-\mathrm{R}_{1}$;

0 l'angle au sommet de $\left(\mathbf{S}_{e}\right)$;

$\mathrm{Q}$ le débit massique, calculé par intégration des $V \cos \alpha$ dans la section considérée;

S la surface de la section;

$V_{m}$ la valeur moyenne de la vitesse dans la section.

Ces lettres seront affectées des indices $e$ ou $s$ suivant que l'on considère les surfaces $\left(\mathbf{S}_{e}\right)$ ou $\left(\mathrm{S}_{8}\right)$. Toutefois, quand aucune confusion n'est possible, nous ne mentionnerons pas ces indices afin d'alléger l'écriture. 


\section{II. - THEOREME D'EULER}

Nous ne reprenảrons pas ici la démonstration de ce théorème, qui dérive directement de la relation $\mathrm{F}=m \gamma$. Nous nous contenterons d'en mentionner un énoncé.

Soit une masse d'eau (M), limitée à l'instant $t$ par une surface fermée $(\Sigma)$; $d Q$ le débit massique traversant un élément $d \sigma$ de cette surface $[d \mathrm{Q}>0$ pour un écoulement de l'extérieur vers l'intérieur de $(\Sigma)], V$ la vitesse de l'eau au centre de $d \sigma$.

En régime permanent, l'ensemble des forces extérieures appliquées à $(\mathbf{M})$ et des vecteurs $\vec{V} d Q$ forme un système équivalent à zéro.

\section{III. - APPLICATION AUX TURBINES A REACTION}

Il est classique de calculer le couple moteur en appliquant ce théorème au volume d'eau compris entre les deux surfaces de révolution $\left(S_{e}\right)$ et $\left(\mathbf{S}_{s}\right)$.

Les forces extérieures appliquées à ce volume d'eau comprennent :

- les forces au contact eau-roue proprement dite, de couple - C par rapport à l'axe ( $\mathrm{C}$ couple moteur);

- les forces au contact eau-pièces fixes, de couple - $\mathrm{C}^{\prime}$;

- les forces de pesanteur et les pressions sur $\left(\mathrm{S}_{e}\right)$ et $\left(\mathrm{S}_{s}\right)$ dont le couple par rapport à l'axe est nul.

$\mathrm{Si}_{e}$ et $\mathrm{C}_{s}$ désignent les couples des vecteurs $\overrightarrow{\mathrm{V}} d \mathrm{Q}$ relatifs aux surfaces $\left(\mathrm{S}_{e}\right)$ et $\left(\mathrm{S}_{s}\right)$, le théorème d'Euler permet d'écrire :

$$
\mathrm{C}+\mathrm{C}^{\prime}=\mathrm{C}_{e}+\mathrm{C}_{s}
$$

Nous conviendrons de considérer comme positif le débit traversant les surfaces $\left(\mathrm{S}_{\epsilon}\right)$ et $\left(\mathrm{S}_{8}\right)$ de l'amont vers l'aval et nous négligerons $\mathrm{C}^{\prime}$ devant C (17), ce qui nous permet d'écrire :

$$
\mathrm{C}=\mathrm{C}_{e}-\mathrm{C}_{3}
$$

Ón en déduit immédiatement les expressions de la puissance fournie à la roue et du rendement :

$$
\begin{aligned}
& \mathrm{W}=\mathrm{NC}=\mathrm{N}\left(\mathrm{C}_{e}-\mathrm{C}_{s}\right) \\
& \eta=\frac{\mathrm{W}}{g \mathrm{QH}_{n}}=\mathrm{E}_{e}-\mathrm{E}_{s}
\end{aligned}
$$

(17) La validité de cette approximation est étudiéc au paragraphe suivant. en posant :

$$
\mathrm{E}_{c}=\frac{\mathrm{NC}_{e}}{g \mathrm{QH}_{n}} \quad \text { et } \mathrm{E}_{s}=\frac{\mathrm{NC}_{s}}{g \mathrm{QH}_{n}}
$$

$\mathrm{E}_{c}$ et $\mathrm{E}_{s}$ sont des coefficients caractéristiques de la rotation de l'eau.

Nous prendrons comme surface $\left(\mathrm{S}_{s}\right)$ une section droite de l'aspirateur. En supposant l'ćcou. lement symétrique et les composantes radiales de la vitesse négligeables, la prospection d'un rayon de la section au tube de Pitot permet aisément le calcul de $\mathrm{E}_{s}$ par l'expression :

$$
\mathrm{E}_{s}=\frac{\mathrm{N}}{g \mathrm{H}_{i l}} \frac{\int_{\mathrm{R}_{1}^{2}}^{\mathrm{R}_{2}{ }^{2}} r \mathrm{~V} \sin \alpha \cdot \mathrm{V} \cos \alpha \cdot d\left(r^{2}\right)}{\int_{\mathrm{R}_{1}{ }^{2}}^{\mathrm{R}_{i^{2}}{ }^{2}} \mathrm{~V} \cos \alpha \cdot d\left(r^{2}\right)}
$$

Donc, connaissant le rendement et le résultat des mesures au tube de Pitot, on peut déterminer $\mathrm{E}_{e}$ et $\mathrm{C}_{e}$.

Nous en déduirons un angle moyen d'entrée : qui, par définition, serait l'angle de la vitesse avec la normale à $\left(S_{t}\right)$ dans un écoulement uniforme de même couple $\mathrm{C}_{o}$ et de même débit que l'écoulement réel (composante méridienne toujours perpendiculaire à $\left(\mathbf{S}_{e}\right)$.

Pour le calcul de cet angle, nous devons distinguer deux cas :

a) La surface $\left(S_{e}\right)$ est cylindrique (étude de la sortie du distributeur dans les Francis el Kaplan classiques).

Pour un ćcoulement uniforme, on a : 
$\mathrm{C}_{e}=r \mathrm{~V}_{m} \sin \varphi \mathrm{Q}=\mathrm{E}_{e} \frac{g \mathrm{QH}_{n}}{\mathrm{~N}}=\left(\mathrm{E}_{s}+\eta\right) \frac{g \mathrm{QH}_{n}}{\mathrm{~N}}$

D'oil :

$$
V_{m} \sin \vartheta=\left(\mathbf{E}_{s}+r_{i}\right) \frac{g \mathrm{H}_{n}}{\mathrm{~N} r}
$$

La composante radiale moyenne est égale à :

$$
\mathrm{V}_{m} \cos \varphi=\frac{\mathrm{Q}}{\frac{\varpi}{g} \cdot 2 \pi r \mathrm{~L}}
$$

D'où :

$$
\operatorname{tg} \varphi=\frac{2 \pi \pi L}{N} \frac{\left(E_{s}+\eta\right) H_{n}}{Q}
$$

On remarque que la valeur de $r$ n'intervient pas dans l'expression de $\operatorname{tg} \varphi$.

Si l'on exprime :

$$
\begin{aligned}
& \mathrm{L} \text { et } \mathrm{H}_{n} \text { en } \mathrm{m} \\
& \mathrm{Q} \text { en } \mathrm{m}^{3} / \mathrm{s} \\
& \mathrm{N} \text { en } \mathrm{tr} / \mathrm{mn} \\
& \mathrm{E}_{s} \text { et } \eta \text { en } \%
\end{aligned}
$$

on obtient :

$$
\operatorname{tg} \varphi=5,89 \frac{\mathrm{L}}{\mathrm{N}} \frac{\left(\mathrm{E}_{s}+\eta\right) \mathrm{H}_{n}}{\mathrm{Q}}
$$

b) La section $\left(\mathrm{S}_{e}\right)$ est conique (ou plane $0=0$ ).

Pour un ćcoulement uniforme:

$$
\begin{aligned}
\mathrm{C}_{0}=\int_{\left(\mathrm{s}_{\mathrm{e}}\right)} r \cdot \mathrm{V}_{m} \sin \varphi \mathrm{dQ} \\
\quad=\int_{\mathrm{I}_{1}}^{\mathrm{L}_{2}} l \sin 0 \cdot \mathrm{V}_{m} \sin \varphi \cdot \frac{\mathrm{Q}}{\mathrm{S}} \cdot 2 \pi l \sin 0 d l
\end{aligned}
$$

avec :

$$
\mathrm{S}=\pi\left(\mathrm{L}_{2}^{2}-\mathrm{L}_{1}^{2}\right) \sin \theta
$$

Le calcul donne :

$$
\mathrm{C}_{c}=\mathrm{V}_{m} \sin \varphi \cdot \frac{\mathrm{Q}}{\mathrm{S}} \cdot \frac{2 \pi}{3} \sin ^{2} \theta\left(\mathrm{L}_{2}{ }^{3}-\mathrm{L}_{1}^{3}\right)
$$

D’où :

$$
\mathrm{V}_{m} \sin \varphi=\left(\mathrm{E}_{s}+\eta\right) \frac{g \mathrm{H}_{n}}{\mathrm{~N}} \frac{3}{2} \frac{\mathrm{L}_{2}{ }^{2}-\mathrm{L}_{1}{ }^{2}}{\sin \theta\left(\mathrm{L}_{2}{ }^{3}-\mathrm{L}_{1}^{3}\right)}
$$

la composante méridienne moyenne de la vitesse [perpendiculaire à $\left.\left(\mathrm{S}_{e}\right)\right]$ est évidemment :

$$
V_{m} \cos \varphi=\frac{Q}{\frac{\pi}{g} \mathrm{~S}}
$$

D'où l'expression de l'angle moyen :

$$
\operatorname{tg} \varphi=\frac{3}{2} \frac{\pi \omega}{\mathrm{N}} \frac{\left(\mathrm{L}_{2}^{2}-\mathrm{L}_{1}^{2}\right)^{2}}{\mathrm{~L}_{2}{ }^{3}-\mathrm{L}_{1}{ }^{3}} \frac{\left(\mathrm{E}_{1}+\eta\right) \mathrm{H}_{n}}{\mathrm{Q}}
$$

On remarque que la valeur de 0 n'intervient pas dans l'expression de $\operatorname{tg} \varphi$.

Si l'on exprime :

$$
\begin{aligned}
\mathrm{L}_{1}, \mathrm{~L}_{2} \text { et } \mathrm{H}_{n} & \text { en } \mathrm{m} \\
\mathrm{Q} & \text { en } \mathrm{m}^{3} / \mathrm{s} \\
\mathrm{N} & \text { en } \mathrm{tr} / \mathrm{mn}
\end{aligned}
$$$$
\mathrm{E}_{s} \text { et } \eta \text { en } \%
$$

on obtient :

$$
\operatorname{tg} \varphi=4,42 \frac{1}{\mathrm{~N}} \frac{\left(\mathrm{L}_{2}^{2}-\mathrm{L}_{1}^{2}\right)^{2}}{\mathrm{~L}_{2}{ }^{3}-\mathrm{L}_{1}{ }^{3}} \frac{\left(\mathrm{E}_{s}+\eta\right) \mathrm{H}_{n}}{\mathrm{Q}}
$$

\section{Remarques.}

$1^{\circ}$ On voit que l'angle géométrique moyen est fonction de $\mathrm{E}_{c} / \mathrm{Q}$ ou $\mathrm{C}_{c} / \mathrm{Q}^{2}$.

$2^{\circ}$ On peut très bien substituer la hauteur de chute brute à la chute nette dans tous les calculs. On pourrait même théoriquement employer une hauteur de référence quelconque pourvu qu'elle soit la même pour $\eta, \mathbf{E}_{e}$ et $\mathbf{E}_{s}$.

\section{IV. - VALIDITE DES SIMPLIFICATIONS ADOPTEES DANS LE PARAGRAPHE PRECEDENT}

\section{Définition de l'angle $\varphi$.}

est l'angle d'un écoulement uniforme ayant même couple $\mathrm{C}_{\epsilon}$ et même débit que l'écoulement

(18) Car, par définition, $\mathrm{E}_{e}=\mathrm{NC}_{e} / g \mathrm{QH}_{n}$. réel. Si ce dernier présente des singularités importantes, il peut exister des écarts non négligeables entre $\varphi$ et certains angles locaux, mais $\varphi$ est la valeur qui définit le mieux l'état moyen de l'entrée. 


\section{2。 Permanence de l'écoulement.}

Le théorème d'Euler suppose que le régime est permanent. Or, dans les turbines hydrauliques, cette propriété n'est vérifiée qu'en moyenne (dans le temps), en raison de la turbulence et parfois des perturbations périodiques liées aux passages des aubes ou des pales. Par suite, il faudrait théoriquement évaluer la moyenne des $\vec{V} d Q$ instantanés et non utiliser les vitesses et débits moyens. Un tel calcul est pratiquement impossible, mais l'erreur commise est le plus souvent tout à fait acceptable. A titre d'exemple, prenons le cas d'une vitesse de direction fixe mais dont la grandeur oscille sinusoïdalement dans le temps avec une amplitude égale à $10 \%$ de sa valeur moyenne : l'erreur commise sur le couple n'est que de $1 \%$.

\section{Couple $\mathbf{C}^{\prime}$ sur les pièces fixes.}

Ce couple $\mathrm{C}^{\prime}$ atteint des valeurs non négligeables dans les labyrinthes des turbines Francis et peut-être sur le manteau de certaines turbines Kaplan ou Francis sans ceinture; en effet, ces pièces ne sont séparées de la roue que par un espace restreint où l'eau est animée d'un important mouvement de rotation, s'accompagnant d'efforts tangentiels dont il faut tenir compte.

Il est difficile d'évaluer $\mathrm{C}^{\prime}$ avec précision. Pour fixer un ordre de grandeur, on peut toutefois considérer que les couples sur les surfaces fixe et mobile en regard sont égaux : le couple de freinage de la roue $C_{f}$ n'apparait pas et l'erreur commise est sensiblement égale aux pertes par «frottements fluides » dans les labyrinthes.

$S i$ une fraction $n$ de l'eau de fuite du labyrinthe supérieur est dérivée par un tuyau d'équilibrage et restituée dans l'aspirateur en aval de la section de mesure, on néglige toujours $\mathrm{C}_{f}$ et de plus la fraction $n$ des pertes par fuite.

Enfin, quand on effectue les mesures assez près de la sortie de la roue, il peut arriver que l'eau du labyrinthe inférieur occupe contre la paroi de l'aspirateur une couronne singulière assez mince pour n'être prise en compte que très partiellement par les relevés expérimentaux; on commet alors une erreur sur l'extrapolation à la paroi, qui dépend du détail de l'écoulement dans cette zone, donc variable suivant la turbine. Cependant, compte tenu dè la technique expérimentale et de la forme des écoulements industriels, on peut estimer qu'en première approximation cette erreur est égale aux pertes totales dans le labyrinthe (fuites et frottements).

En conclusion, nous retiendrons que l'application du théorème d'Euler, exposée ci-dessus, fournit une valeur $\eta$ du rendement comprise entre le rendement hydraulique $\eta_{H}$ de la roue proprement dite (pertes labyrinthes et mécaniques exclues) et le rendement $\eta_{\mathrm{T}}$ de l'ensemble de la turbine (pertes mécaniques seules exclues). En pratique, $\eta$ est généralement très voisin de $\eta_{\Pi \pi}$ : nous confondrons ces deux valeurs, une telle approximation étant largement justifiée pour le calcul de l'angle moyen d'entrée.

\section{V. - UTILISATION DES RESULTATS D'UNE PROSPECTION DIRECTE DE L'ECOULEMENT ENTRE DISTRIBUTEUR ET ROUE}

On peut évaluer directement l'angle moyen d'entrée si l'on sait réaliser expérimentalement une prospection de l'écoulement suivant une génératrice de la surface $\left(S_{e}\right)$, en supposant toujours que la composante méridienne de la vitesse est pratiquement perpendiculaire à $\left(\mathrm{S}_{e}\right)$ et que l'écoulement est de révolution autour de l'axe de la turbine).

a) La surface $\left(\mathrm{S}_{e}\right)$ est cylindrique.

On obtient immédiatement :

$$
\begin{aligned}
\mathrm{C}_{e}=\int_{0}^{\mathrm{I}} r \mathrm{~V} & \sin \alpha \cdot \frac{\varpi}{g} \cdot \mathrm{V} \cos \alpha \cdot 2 \pi r d l \\
& =2 \pi r^{2} \frac{\omega}{g} \int_{0}^{\mathrm{L}} \mathrm{V} \sin \alpha \mathrm{V} \cos \alpha d l
\end{aligned}
$$

et :

$$
\mathrm{Q}_{e}=\int_{0}^{\mathrm{L}} \frac{\pi}{g} \mathrm{~V} \cos \alpha \cdot 2 \pi r d l=2 \pi r \frac{\omega}{g} \int_{0}^{\mathrm{L}} \mathrm{V} \cos x d l
$$

D'où :

$$
\begin{gathered}
\mathrm{V}_{m} \sin \varphi=\frac{\mathrm{C}_{e}}{r \mathrm{Q}_{e}}=\frac{\int_{0}^{\mathrm{L}} \mathrm{V} \sin \alpha \cdot \mathrm{V} \cos \alpha d l}{\int_{0}^{\mathrm{I}} \mathrm{V} \cos \alpha d l} \\
\mathrm{~V}_{\mathrm{m}} \cos \varphi=\frac{\mathrm{Q}_{i}}{\frac{\omega}{g} \cdot 2 \pi r \mathrm{~L}}=\frac{l}{\mathrm{~L}} \int_{0}^{\mathrm{L}} \mathrm{V} \cos \alpha d l
\end{gathered}
$$

et :

$$
\operatorname{tg} \varphi=\mathrm{L} \frac{\int_{0}^{\mathrm{I}} \mathrm{V} \sin \alpha \mathrm{V} \cos \alpha d l}{\left[\int_{0}^{\mathrm{L}} \mathrm{V} \cos \alpha \cdot d l\right]^{2}}
$$


b) La surface $\left(\mathrm{S}_{e}\right)$ est plane ou conique (cas général).

Dans ce cas :

$$
\begin{gathered}
\mathrm{C}_{\theta}=\int_{\mathrm{L}_{1}}^{\mathrm{L}_{2}} l \sin \theta \mathrm{V} \sin \alpha \cdot \frac{\pi}{g} \mathrm{~V} \cos \alpha \cdot 2 \pi l \sin \theta d l \\
=2 \pi \sin ^{2} \theta \frac{\pi}{g} \int_{\mathrm{L}_{1}}^{\mathrm{L}_{2}} l^{2} \mathrm{~V} \sin \alpha \cdot \mathrm{V} \cos \alpha \cdot d l \\
\mathrm{Q}_{e}=\int_{\mathrm{L}_{\mathrm{l}}}^{\mathrm{L}_{2}} \frac{\pi}{g} \mathrm{~V} \cos \alpha \cdot 2 \pi l \sin \theta d l \\
=2 \pi \sin \theta \cdot \frac{\pi}{g} \int_{\mathrm{L}_{1}}^{\mathrm{I}_{2}} l \mathrm{~V} \cos \alpha d l
\end{gathered}
$$

On en tire :

$$
\begin{gathered}
\mathrm{V}_{m} \sin \varphi=\frac{\mathrm{C}_{e}}{\mathrm{Q}_{e}} \frac{3}{2} \frac{\mathrm{L}_{2}{ }^{2}-\mathrm{L}_{1}{ }^{2}}{\left(\mathrm{~L}_{2}{ }^{3}-\mathrm{L}_{1}{ }^{3}\right) \sin \theta} \\
=\frac{3}{2} \frac{\mathrm{L}_{2}{ }^{2}-\mathrm{L}_{1}{ }^{2}}{\mathrm{~L}_{2}{ }^{3} \cdot-\mathrm{L}_{1}{ }^{3}}, \frac{\int_{\mathrm{L}_{1}}^{\mathrm{L}_{2}} l^{2} \mathrm{~V} \sin \alpha \mathrm{V} \cos \alpha d l}{\int_{\mathrm{L}_{1}}^{\mathrm{L}_{2}} I \mathrm{~V} \cos \alpha d l} \\
\mathrm{~V}_{m} \cos \varphi=\frac{2}{\frac{\varpi}{g} \pi\left(\mathrm{L}_{2}{ }^{2}-\mathrm{L}_{1}{ }^{2}\right) \sin \theta}=\frac{\int_{\mathrm{L}_{1}}^{\mathrm{L}_{2}} I \mathrm{~V} \cos \alpha d l}{\mathrm{~L}_{2}{ }^{2}-\mathrm{L}_{1}{ }^{2}}
\end{gathered}
$$

et :

$$
\operatorname{tg} \varphi=\frac{3}{4} \frac{\left(\mathrm{L}_{2}^{2}-\mathrm{L}_{1}{ }^{2}\right)^{2}}{\mathrm{~L}_{2}{ }^{3}-\mathrm{L}_{1}{ }^{3}} \frac{\int_{\mathrm{L}_{1}}^{\mathrm{L}_{\mathrm{L}_{2}}} l^{2} \mathrm{~V} \sin \alpha \cdot \mathrm{V} \cos \alpha \cdot d l}{\left[\int_{\mathrm{r}_{1}}^{\mathrm{I}_{\alpha_{2}}} I \mathrm{~V} \cos \alpha d l\right]^{2}}
$$

\section{Remarque.}

Dans tous les cas, la valeur absolue de la vitesse $V$ intervient d'une manière homogène au numérateur et au dénominateur. Aussi peut-on se contenter de sa connaissance à un coefficient multiplicatif près (tarage de l'appareil de mesure par exemple).

\section{Application de la notion d'angle moyen d'en- trée.}

En l'absence de mesures entre distributeur et roue, les valeurs de $\bullet$ seront surtout destinées à l'étude des triangles de vitesses à l'entrée, du guidage des directrices et de la réaction de l'écoulement à certaines transformations de la turbine (variation de chute, changement de roue par exemple).

Si l'on dispose des résultats de prospection à l'amont de la roue, on pourra, soit mettre en évidence d'éventuelles anomalies (erreur de mesure ou dissymétries d'écoulement), soit calculer le rendement si ce dernier est ignoré. Dans ce cas, on peut théoriquement se dispenser de passer par l'intermédiaire de $\varphi$ pour appliquer le théorème d'Euler. Mais, en pratique, son introduction sera en général justifiée par certaines de ses propriétés (en particulier indépendance du tarage en vitesse de la sonde utilisée à l'amont, conservation de $\varphi$ pour des variations de chute ou de position des pales, du moins en première approximation et dans certaines limites).
M. le Président remercie M. Campuas et ouvre la discussion.

Mr. Hug voudrait développer un point qui est peut-être passé inaperçu dans la communication de M. Campnas :

Personne ne contestera l'utilité du Pitot pour donner des angles et des répartitions de vitesses relatives. Ce qu'il $y$ a de remarquable dans le travail de M. CAmpmas, c'est qu'il arrive, avec une précision comparable à celle des autres méthodes traditionnelles de mesure de rendement, à des déterminations absolues.

M. Hug souhaiterait que M. Campmas veuille bien développer ici les techniques de ces mesures de Pitot, en particulier en ce qui concerne les problèmes d'étalonnage, risque de vibration et influence de la turbulence de l'écoulement.

M. Campmas indique que, sous sa forme actuelle, le tube de Pitot utilisé ne nécessite pas un étalonnage préalable (du moins dans sa plage d'utilisation normalement comprise entre 5 et $15 \mathrm{~m} / \mathrm{s}$ ).

D'autre part, l'expérience montre que, pour les écoulements industriellement rencontrés, la turbulence ne paraît pas causer d'erreur importante. En effet, c'est sur les Francis que le phénomène est a priori le plus à craindre. Or, on constate dans les essais recents un bon recoupement entre les débits déduits du tube de Pitot et les débits mesurés par d'autres méthodes, moulinets et thermodynamique en particulier (après introduction, il est vrai, de termes correctifs parfois importants destinés à tenir compte de la présence de poches ou de torches de gaz à demi-charge et à pleine charge).

Enfin l'amplitude des vibrations a été réduite par toute une série de détails technologiques (dimensions et rigidité de la tige-support, conception du guide, etc.). Pour l'instant, nous n'avons décelé aucune. singularité liée à l'enfoncement du tube de Pitot, qui pourtant modifie le 
régime de vibrations et peut notamment faire apparaître des résonances locales.

M. Hug voudrait savoir comment M. CAMPMas arrive à des précisions aussi grandes alors qu'une communication de $M$. Winternitz donne des écarts de plus de $30 \%$ lorsque le tube vibre et que d'autres travaux, non públiés, indiquent des valeurs encore plus fortes.

En réponse, M. Campmas fait remarquer que la précision dépend dans de très larges limites des conditions expérimentales : même avec l'appareillage actuellement en service, il connaît des cas d'erreurs très importantes (certaines. marches en déchargeur de Kaplans, zone centrale de l'aspirateur des Francis à faible charge). Mais il s'agit là de cas exceptionnels dans la pratique et, c'est un fait d'expérience, les écoulements industriels sous les roues (d'ailleurs très semblables pour toutes les turbines Francis ou Kaplan quels que soient les constructeurs) peuvent être étudiés avec un tube de Pitot.

Remarquons bien que nous ne prétendons pas atteindre une précision de 1 à $2 \%$ (d'ailleurs résultant d'une moyenne de 10 à 20 mesures indépendantes) dans tous les cas; nous constatons seulement qu'on peut l'obtenir dans certains cas et nous prévoyons une étude pour mieux définir les possibilités et les limites de la méthode.

M. FErry ajoute que la prospection par tube de Pitot associé à un élément détecteur de direction de la vitesse, telle que la pratique l'équipe de M. Campmas, se révèle comme particulièrement intéressante pour la mesure du débit dans le cas des vitesses fortement obliques par rapport à l'axe général de l'écoulement, lorsqu'on peut, a priori, négliger la composante de la vitesse suivant l'axe de rotation de la sonde. Dans ce cas, l'élément détecteur de direction de la vitesse donne cette direction avec une excellente précision; l'erreur provenant de la mauvaise connaissance de cette orientation est alors bien plus faible que dans l'exploration par moulinets. Reste l'erreur due aux fluctuations de la grandeur et de l'orientation de la vitesse. Le tube de Pitot proprement dit donne pratiquement la moyenne quadratique de la grandeur de la vitesse vraie, à peu près indépendamment de l'angle de celle-ci avec sa direction moyenne. Compte tenu de ce fait, on peut voir que les fluctuations modérées n'entraînent que de très faibles erreurs.

\section{Duport fait remarquer :}

$1^{\circ}$ Que la roue, installée dans le groupe bulbe dont a parlé M. Campmas, n'avait pas été spécialement tracée pour un groupe bulbe. La proportion des pertes dans la roue par rapport aux pertes totales serait plus faible si on avait utilisé une roue spécialement tracée pour le cadre bulbe;

$2^{\circ}$ Que la méthode devrait s'appliquer moins bien aux Kaplans qu'aux bulbes, car, dans les turbines Kaplan, la régularité de répartition de vitesse et de circulation, à l'entrée comme à la sortie de la roue, sera moins bonne que dans les bulbes, de même que la symétrie de révolution $\mathrm{y}$ sera moins rigoureuse.

En réponse à la remarque précédente, M. Campmas indique qu'un essai récent, effectué sur une Kaplan classique, a donné entière satisfaction. Toutefois, il est possible que certaines analyses détaillées soient plus difficiles sur ce type de machines.

M. Langlors, en complément d'une des remarques qui viennent d'être présentées par M. Duport, insiste sur le fait que la séparation des pertes totales, effectuée par M. Campmas sur le groupe de Beaumont-Monteux, met en évidence la part prépondérante des pertes dans la roue d'une part, et la faiblesse des pertes dans l'aspirateur par rapport à une turbine classique d'autre part.

Il est bon d'ajouter d'ailleurs que, tout en restant prépondérantes, les pertes dans la roue auraient pu être quelque peu inférieures si celle-ci avait été étudiée spé- cialement, ce qui n'était pas le cas à Beaumont-Monteux; quoi qu'il en soit, le rendement optimal obtenu, qui semble être de l'ordre de 89 à $90 \%$, est très satisfaisant et permet d'espérer normalement un gain de 2 à 3 points par rapport à un groupe de type classique.

A une question de M. Duport, M. Campmas répond qu'il y a un écart de $2 \%$ entre la mesure industrielle et la mesure sur modèle dans la zone du rendement maximal.

M. le Président demande ce que pensent les constructeurs des centrales, plus que ceux des turbines, de la suggestion, faite par M. Campmas, de ménager des orifices d'accès de façon à faciliter les mesures ultérieures :

M., Langlors ne voit pas d'inconvénient à prévoir dans les projets d'équipement de groupes bulbes toutes facilités pour l'installation de l'appareillage qui permettra d'effectuer les mesures suggérées par M. Campmas.

M. LANGLors fait remarquer que les dispositions à prendre sont d'importance modeste et se trouvent, au surplus, analogues à celles qui ont été adoptées il y a quelques années pour les groupes à axe vertical en prévoyant notamment, lors de la construction, les orifices nécessaires à l'installation des dispositifs piézométriques. A l'image des piézomètres, les dispositifs dont il s'agit permettront un contrôle systematique des rendements en cours d'exploitation et même de suivre l'évolution de la séparation des pertes.

M. Lugrez, répondant à M. le Président, indique qu'il n'est d'ailleurs pas indispensable de prévoir les dispositions nécessaires à ces essais sur toutes les machines du même type; il suffirait d'équiper un nombre réduit de turbines pour déterminer leurs performances à la mise en service et suivre celles-ci en cours d'exploitation.

M. le Président dit que M. Campmas est allé un peu plus loin : il avait dit qu'il faudrait prendre le cas de l'incident. $A$ priori, on ne sait pas sur quelle machine aura lieu l'incident.

M. Campmas indique qu'il désirerait disposer d'un contrôle sur au moins un groupe de chaque type, mais qu'il n'est pas nécessaire d'équiper tous les groupes s'ils sont rigoureusement identiques.

M. Duporr dit qu'il y a un grand intérêt à faire l'analyse des pertes par pitotage, parce que c'est la seule méthode qui risque d'aboutir un jour pour élucider le fameux problème des effets d'échelle de rendement; on peut faire simultanément cette méthode sur modèle et sur prototype. Elle est supérieure à la méthode statistique, employée depuis très longtemps, mais qui ne donne qu'un résultat global, sans aucune possibilité d'analyse.

M. NARCy indique que le problème de ménager des emplacements pour un tube de Pitot dans les installations actuelles ne s'est pas posé jusqu'ici; il s'est posé seulement pour des Francis pour analyser la torche. Pour les turbines, les dispositions à prendre sont très simples. Dans certaines grosses Kaplans, il y aura un petit bout de galerie à ménager autour de l'aspirateur. Mais il $y$ a une chose insurmontable: pereer $4 \mathrm{~m}$ de béton pour aller jusqu'à l'aspirateur.

M. Langlors dit qu'en vue de permettre un contrôle efficace de la méthode suggérée par M. Campmas, il propose que des essais de séparation des pertes soient effectués sur des groupes hydrauliquement similaires appartenant aux types axial et classique (Francis ou Kaplan). Installer d'avance des petits dispositifs pour permettre à M. CAMpMas de faire ses mesures n'entrấnera pas de complications très grandes. Plus la machine est grande, plus ce sera facile.

M. le Président constate que tout le monde est d'accord pour dire qu'il est utile, au moins sur un certain nombre de machines, de décider, avant la conception, yu'on 
aménagerait les emplacements nécessaires à faciliter les mesures ultérieures.

M. Chevalier pose les questions suivantes :

En admettant qu'il soit possible, sur une turbine Kaplan à axe vertical, de faire des mesures à l'amont et à l'aval de la roue et que l'on ne puisse atteindre le rendement par une méthode globale, est-ce que la méthode que M. Campmas a exposée s'appliquerait à des régimes voisins de la déconjugaison?

En effet, dans des régimes de déconjugaison, pour lesquels le rendement chute de 3 ou 4 points, on a observé sur modèle, grâce à des sondes bidirectionnelles, des vitesses radiales non négligeables (angle atteignant une dizaine de degrés). Est-ce que, dans ce cas, la comparaison des angles moyens d'entrée et de sortie reste un critère valable en ce qui concerne la valeur absolue de la méthode?

M. Campmas répond que, pour l'instant, il n'envisage pas l'utilisation des rendements déduits des mesures au tube de Pitot si tout recoupement avec une autre méthode est impossible; en début de discussion, il a signalé qu'une étude était prévue pour déterminer les possibilités et les limites d'emploi de la méthode.

D'autre part, s'il existe une composante radiale correspondant à un angle de 10 degrés de la vitesse sur le rayon, on commet évidemment une erreur (égale à $\cos 10^{\circ}$ ) qu'un contrôle, soit avec une autre méthode (même relative), soit avec les angles d'entrée, permet de déceler. Il faut ajouter que, si la pratique le justifie, l'appareillage pourra être modifié pour permettre la mesure de cette composante radiale.

M. FERry ajoute que lorsqu'on trace la colline de renciement, angle du distributeur, angle des pales, il importe assez peu que la colline soit déformée lorsqu'on s'écarte de la conjugaison optimale; ce que l'on cherche, c'est la zone optimale; il faut et il suffit donc que la crête de la colline soit donnée correctement.

M. le Président dit que la discussion a été abondante et son sujet plus général que celui du groupe bulbe qui, dans l'exposé, était là comme support.

II. le Président remercie et félicite M. Campmas de sa communication.

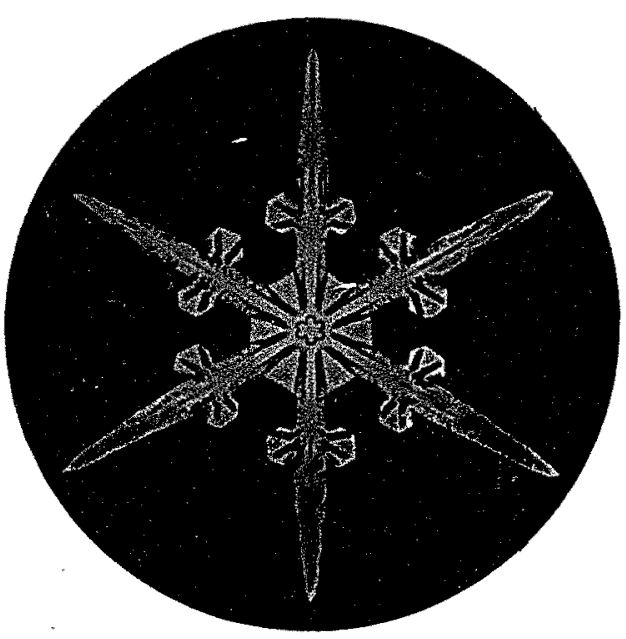

